\title{
Bridging the Gap: A Reticulo-Propriospinal Detour Bypassing an Incomplete Spinal Cord Injury
}

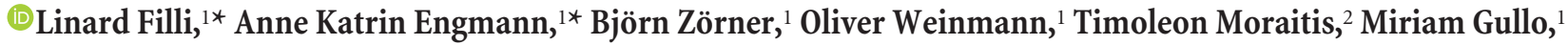 \\ Hansjörg Kasper, ${ }^{1}$ Regula Schneider, ${ }^{1}$ and Martin E. Schwab ${ }^{1}$ \\ ${ }^{1}$ Brain Research Institute, University of Zurich and Department of Health Sciences and Technology, ETH Zurich, 8057 Zurich, Switzerland, and ${ }^{2}$ Institute of \\ Neuroinformatics, University and ETH Zurich, 8057 Zurich, Switzerland
}

\begin{abstract}
Anatomically incomplete spinal cord injuries are often followed by considerable functional recovery in patients and animal models, largely because of processes of neuronal plasticity. In contrast to the corticospinal system, where sprouting of fibers and rearrangements of circuits in response to lesions have been well studied, structural adaptations within descending brainstem pathways and intraspinal networks are poorly investigated, despite the recognized physiological significance of these systems across species. In the present study, spontaneous neuroanatomical plasticity of severed bulbospinal systems and propriospinal neurons was investigated following unilateral C4 spinal hemisection in adult rats. Injection of retrograde tracer into the ipsilesional segments $\mathrm{C} 3$ - $\mathrm{C} 4$ revealed a specific increase in the projection from the ipsilesional gigantocellular reticular nucleus in response to the injury. Substantial regenerative fiber sprouting of reticulospinal axons above the injury site was demonstrated by anterograde tracing. Regrowing reticulospinal fibers exhibited excitatory, vGLUT2-positive varicosities, indicating their synaptic integration into spinal networks. Reticulospinal fibers formed close appositions onto descending, double-midline crossing $\mathrm{C} 3$-C4 propriospinal neurons, which crossed the lesion site in the intact half of the spinal cord and recrossed to the denervated cervical hemicord below the injury. These propriospinal projections around the lesion were significantly enhanced after injury. Our results suggest that severed reticulospinal fibers, which are part of the phylogenetically oldest motor command system, spontaneously arborize and form contacts onto a plastic propriospinal relay, thereby bypassing the lesion. These rearrangements were accompanied by substantial locomotor recovery, implying a potential physiological relevance of the detour in restoration of motor function after spinal injury.
\end{abstract}

Key words: locomotion; neuroanatomical plasticity; propriospinal; reticulospinal; spinal cord injury

\section{Introduction}

Although spontaneous long-distance regeneration is absent in the adult mammalian CNS, patients and animal models of spinal cord injury (SCI) characteristically show some degree of functional recovery after incomplete SCI (Tator et al., 1995). These functional improvements are attributed to plastic adaptations within the CNS occurring on the molecular and cellular level, but also on the level of entire neural circuits (Zörner and Schwab,

Received Feb. 19, 2014; revised Aug. 18, 2014; accepted Aug. 24, 2014.

Author contributions: L.F., A.K.E., B.Z., and M.E.S. designed research; L.F., A.K.E., B.Z., O.W., T.M., M.G., R.S., and H.K. performed research; L.F. and A.K.E. analyzed data; L.F., A.K.E., and M.E.S. wrote the paper.

The authors' laboratory was supported by Swiss National Science Foundation Grant 31003A-149315-1, the National Centre for Competence in Research "Neural Plasticity and Repair" of the Swiss National Science Foundation, the Spinal Cord Research Consortium of the Christopher and Dana Reeve Foundation (Springfield, NJ), and the European Union advanced ERC Grant "Nogorise." We thank our colleagues Lorne Mendell (Stony Brook University), Eric Frank (Tufts University), and Jean-Marc Fritschy and Arko Ghosh (University and ETH Zurich) for their valuable scientific and experimental input; and Alexander van der Bourg and the Center for Microscopy and Image Analysis of the University Zurich for their methodological support.

The authors declare no competing financial interests.

*L.F. and A.K.E. contributed equally to this work.

Correspondence should be addressed to Dr. Linard Filli, Department of Neurology, University Hospital Zurich, Frauenklinikstrasse 26,8091 Zurich, Switzerland. E-mail: linardfilli@hotmail.com or linard.filli@usz.ch.

L. Filli's present address: Department of Neurology, University Hospital Zurich, 8091 Zurich, Switzerland.

B. Zörner's present address: Department of Neurology, University Hospital Zurich, 8091 Zurich, Switzerland.

DOI:10.1523/JNEUROSCI.0701-14.2014

Copyright $\odot 2014$ the authors $\quad 0270-6474 / 14 / 3413399-12 \$ 15.00 / 0$
2010). Neuroanatomical plasticity subsequent to partial SCI comprises sprouting of spared fibers (compensatory plasticity), sprouting and regrowth of severed fibers (regenerative plasticity), and adaptations within supraspinal and intraspinal systems. Spontaneous anatomical plasticity after SCI has mainly been studied for the corticospinal system and was frequently associated with functional recovery (Rosenzweig et al., 2010). Anatomical plasticity within brainstem and intraspinal systems is much less investigated, although the crucial role of these systems in controlling basic motor functions, such as locomotion, is well known in rodents, cats, and primates (Lawrence and Kuypers, 1968; Shik and Orlovsky, 1976).

The medial reticular formation of the brainstem, specifically the nucleus reticularis gigantocellularis (NRG), is a key unit integrating locomotor commands from the mesencephalic locomotor region (MLR), from the tectum and tegmentum, and from cerebellar and cerebral centers (Alstermark et al., 1992). The NRG produces essential outputs to spinal locomotor networks: Thermal inactivation of the NRG blocked MLR-induced locomotion in the cat (Noga et al., 2003), whereas electrical and pharmacological stimulation of the same region elicited locomotion in decerebrated rats (Kinjo et al., 1990). Ballermann and Fouad (2006) revealed the remarkable capacity of spared reticulospinal fibers for spontaneous compensatory sprouting upon incomplete 
SCI in rats, accompanied by significant locomotor recovery. In contrast, virtually nothing is known about spontaneous regrowth of severed reticulospinal fibers in higher mammals.

Plasticity within propriospinal networks after SCI is still poorly investigated, presumably because of their anatomical and physiological complexity. Intraspinal neurons play an essential role in integrating convergent motor and sensory inputs and in modulating the activity of spinal circuits to produce accurate outputs, as shown for locomotion (Gerasimenko et al., 2008) and the $\mathrm{C} 3-\mathrm{C} 4$ propriospinal system in cats and primates (Alstermark et al., 2007). The few studies on neuroplasticity of propriospinal neurons (PSNs) after SCI imply a high intrinsic potential of these neurons for neuroanatomical adaptations (Flynn et al., 2011). Especially the formation of propriospinal detour pathways circumducting supraspinal commands around spinal lesions could be an important substrate for functional recovery (Bareyre et al., 2004; Courtine et al., 2008; van den Brand et al., 2012). So far, spontaneous formation of propriospinal relays has only been specified for the corticospinal system, but not for bulbospinal systems, which have major locomotor relevance in animals.

In contrast to previous studies examining compensatory plasticity of unlesioned reticulospinal fibers (Ballermann and Fouad, 2006; Zörner et al., 2014), this study aimed to analyze neuroanatomical adaptations of severed NRG projections and propriospinal fibers upon incomplete SCI in rats using combined anterograde and retrograde neuroanatomical tracings and immunohistochemical stainings. Recovery of locomotor function was assessed by detailed kinematic analysis.

\section{Materials and Methods}

Animals

All experimental procedures were performed in female adult Lewis rats (180-220 g, Centre d'Elevage Janvier). Animals were housed in groups of 3 or 4 subjects per cage, at a 12:12 h light:dark cycle, with water and food provided ad libitum. Animals were given at least $7 \mathrm{~d}$ of acclimatization before the onset of any experimental procedure. All experiments were approved by the Veterinary Office of the Canton Zurich, Zurich, Switzerland.

\section{Experimental groups}

The design for the neuroanatomical tracing in all subexperiments is shown in Figure $1 a$. Three animal groups were investigated for plastic rearrangements following SCI: intact animals, animals traced $3 \mathrm{~d}$ post injury (dpi; perfused $17 \mathrm{dpi}$ ), and animals traced 29 dpi (perfused 43 dpi). Injections of the neuroanatomical tracer mini-emerald (ME) at spinal levels C3-C4 were examined in two different ways: the retrograde transport of the tracer was used to analyze axonal fiber sprouting of descending brainstem systems rostral to the lesion site. Increased numbers of retrogradely labeled neurons were taken as indirect measure of axonal sprouting above the SCI (see Fig. 1): intact animals $(n=6)$, animals perfused $17 \mathrm{dpi}(n=8)$, and animals perfused $43 \mathrm{dpi}(n=6)$. Anterograde transport of the same tracer was used to investigate anatomical plasticity of descending C3-C4 propriospinal fibers (see Fig. 5): animals perfused $17 \mathrm{dpi}(n=8)$ and animals perfused 43 dpi $(n=6)$. By assuring lesion completeness in animals $17 \mathrm{dpi}$ and $43 \mathrm{dpi}$ (see Analysis of lesion completeness), we could exclude C3-C4 PSN projections directly descending in the ipsilesional white matter to the cervical enlargement (which is not possible in intact animals). Thus, any labeled PSN fiber in the ipsilesional cervical enlargement must have crossed the spinal cord midline twice: once above the injury to the contralesional spinal cord and once in the cervical enlargement back to the ipsilesional gray matter. Regenerative sprouting of severed reticulospinal fibers was investigated by anterograde tracer injections (ME) into the ipsilesional NRG (see Fig. $2)$ : intact animals $(n=8)$, animals perfused $43 \mathrm{dpi}(n=7)$. The same animals were traced with the retrograde red-fluorescent dextran (TMR) at spinal levels $\mathrm{C} 6-\mathrm{C} 8$ to visualize the cell bodies of double-crossing propriospinal neurons rostral to the injury (see Fig. 4). Kinematic assessment of locomotor performance was performed weekly until 4 weeks post injury (see Fig. $6 ; n=6$ animals).

\section{Spinal cord injury}

Surgical procedures (spinal lesion, neuroanatomical tracing) were performed in deeply anesthetized animals (Hypnorm, $0.6 \mathrm{ml} / \mathrm{kg}$ body weight, Janssen Pharmaceutics; Dormicum, $3.75 \mathrm{mg} / \mathrm{kg}$ body weight, Roche Pharmaceuticals). SCI was performed as described previously (Zörner et al., 2010). Briefly, after skin incision, muscles of the neck and upper back were separated and the vertebral laminae C3-C5 were cleared from connective tissue. A C4 laminectomy was performed, and the dura was carefully removed. A complete, right-sided hemisection at cervical spinal level C4 (sparing C4 dorsal and ventral roots) was performed by repeated transection using a sharp sapphire knife (World Precision Instruments). Animals were sutured and received analgesics (Rimadyl, 2.5 $\mathrm{mg} / \mathrm{kg}$ body weight, Pfizer) for $2 \mathrm{~d}$, and antibiotics (Baytril, $5 \mathrm{mg} / \mathrm{kg}$ body weight, Bayer) for 1 week after surgery. Subcutaneous injections of Glucose-Ringer Solution (Fresenius Medical Care) were given to prevent dehydration after surgery. Manual micturition was performed twice a day, until normal bladder control had returned.

\section{Neuroanatomical tracing}

After laminectomy/craniectomy, the dura was carefully removed to expose the region of interest in the spinal cord/brain. Tracer injections were performed using a stereotaxic apparatus (David Kopf Instruments) with a precision of $10 \mu \mathrm{m}$. A Nanofil syringe (Hamilton) connected to a 35 -gauge needle was used to stereotactically inject the neuroanatomical tracers at the target region with a constant velocity of $6 \mathrm{nl} / \mathrm{s}$ (World Precision Instruments). Green-fluorescent ME (10,000 MW dextran tagged with fluorescin and biotin: Invitrogen) and a red-fluorescent dextran (TMR, 3000 MW dextran tagged with tetramethylrhodamine: Invitrogen) were used as neuroanatomical tracers, both of them injected as $10 \%(\mathrm{w} / \mathrm{v})$ solutions, diluted in sterile $\mathrm{H}_{2} \mathrm{O}$. After injection of the tracer, the needle was kept in position for $3 \mathrm{~min}$, allowing the tracer to penetrate into the tissue.

Tracer injections into the ipsilesional C3-C4 gray matter. Four ME injections with a volume of $100 \mathrm{nl}$ each were performed in the ipsilesional spinal segments C3-C4: coordinates, mediolateral (ML), $900 \mu \mathrm{m}$ from spinal cord midline; dorsoventral (DV), $1600 \mu \mathrm{m}$ from dorsal surface. The injections were separated by $500 \mu \mathrm{m}$ in the anteroposterior (AP) direction. Tracer injections were performed in intact animals $(n=6)$, animals 3 dpi $(n=8)$, and animals $29 \mathrm{dpi}(n=6)$. All animals were killed $14 \mathrm{~d}$ after tracer injections (17 dpi and $43 \mathrm{dpi}$ ).

Tracer injections into the ipsilesional cervical enlargement (C6-C8). Ten ipsilesional TMR injections with a volume of $225 \mathrm{nl}$ each were performed in the ipsilesional spinal segments C6-C8 (coordinates: ML, $900 \mu \mathrm{m}$; $\mathrm{DV}, 1600 \mu \mathrm{m}$ ). Injections were separated by $500 \mu \mathrm{m}$ in the AP direction. Tracings were performed $29 \mathrm{dpi}(n=7)$, and animals were killed $14 \mathrm{~d}$ thereafter (43 dpi).

Tracing of reticulospinal fibers. After craniectomy, the dura was locally opened and $50 \mathrm{nl} \mathrm{ME}$ was injected into the ipsilesional NRG (coordinates: AP, $4600 \mu \mathrm{m}$ caudal from $\lambda$; ML, $1050 \mu \mathrm{m}$ lateral from $\lambda$; DV, 7800 $\mu \mathrm{m}$ from brain surface). Tracer injections were performed in intact animals $(n=8)$ and animals 29 dpi $(n=7)$. Animals were killed for histological analysis $14 \mathrm{~d}$ later (43 dpi).

\section{Tissue processing}

Animals were transcardially perfused with $50 \mathrm{ml}$ of a $1 \%$ Heparin-Ringer solution (B. Brown Medical) followed by $300 \mathrm{ml}$ of a 4\% PFA (SigmaAldrich) solution comprising $5 \%$ sucrose. The brain and spinal cord were dissected, postfixed in $4 \% \mathrm{PFA}$ at $4^{\circ} \mathrm{C}$ for $24 \mathrm{~h}$, and transferred into a $30 \%$ sucrose solution at $4^{\circ} \mathrm{C}$ for $3 \mathrm{~d}$ for cryoprotection. The tissue was embedded in Tissue-Tek OCT compound, frozen, and cut in $40-\mu \mathrm{m}$-thick brain and spinal cord cross-sections. Free-floating sections (for immunohistochemical staining) were collected in $4^{\circ} \mathrm{C}$ cold $0.1 \mathrm{M}$ phosphate buffer and kept in anti-freeze solution (15\% sucrose, $30 \%$ ethylene glycol in $50 \mathrm{~mm}$ phosphate buffer) at $-20^{\circ} \mathrm{C}$ until further processing. 


\section{Analysis of lesion completeness}

For the accurate analysis of lesion size, spinal cord cross-sections were stained with a cresyl violet solution (Nissl-stain; for details see Kapitza et al., 2012). Rats with incomplete lesions or overhemisections, including secondary damage, were strictly excluded from the study. Fiber tracings of ascending and descending tracts were additionally used to confirm lesion completeness. The absence of ascending fibers in the ipsilesional C3-C4 white matter after retrograde C6-C8 tracing, or the absence of descending fibers in the $\mathrm{C} 5$ white matter after anterograde C3-C4 tracings were taken as measure of complete unilateral spinal cord transection.

\section{Immunohistochemistry}

For vGLUT2/ME costainings (see Fig. 3e,f), free-floating spinal cord cross-sections were permeabilized in TNB $(0.5 \%$ TopBlock; LuBioScience in $0.1 \mathrm{M}$ Tris) containing $5 \%$ normal goat serum and $0.4 \%$ Triton $\mathrm{X}-100$ for $30 \mathrm{~min}$, subsequently washed in PBS, and incubated with the primary antibody (rabbit-anti-vGLUT2 1:750, Synaptic Systems, RRID: AB_887883) diluted in hypotonic $0.01 \mathrm{M}$ phosphate buffer (PB) containing $0.1 \%$ Triton $\mathrm{X}-100$ and $2 \%$ normal goat serum at $4^{\circ} \mathrm{C}$ for $3 \mathrm{~d}$. The sections were then washed, blocked with TNB for $10 \mathrm{~min}$, and incubated with the fluorescently labeled secondary antibody (DyLight649 goatanti-rabbit IgG 1:200, Jackson ImmunoResearch Laboratories) and a fluorescently labeled Streptavidin (DyLight488-Streptavidin 1:1000, Jackson ImmunoResearch Laboratories) to amplify the ME signal at $4^{\circ} \mathrm{C}$ overnight. Secondary antibodies were diluted in hypotonic $0.01 \mathrm{~m} \mathrm{~PB}$ containing $0.1 \%$ Triton $\mathrm{X}-100$ and $2 \%$ normal goat serum.

For the quadruple stainings (ME/vGLUT1/vGLUT2/vGAT; see Fig. $3 a-c)$, free-floating spinal cord cross-sections were washed in $0.1 \mathrm{M} \mathrm{PB}$ before blocking and permeabilizing them in $0.01 \mathrm{M} \mathrm{PB}$ containing $0.3 \%$ Triton X-100 and 5\% normal goat serum for $10 \mathrm{~min}$ and subsequently in $0.01 \mathrm{M} \mathrm{PB}$ containing $0.05 \%$ Triton X-100 and $5 \%$ normal goat serum for $20 \mathrm{~min}$. The sections were washed in $50 \mathrm{~mm}$ Tris-glycine and $50 \mathrm{~mm}$ $\mathrm{NH}_{4} \mathrm{Cl}$ solution containing $2 \%$ normal goat serum for 20 min to reduce background levels. After washing in $0.01 \mathrm{M} \mathrm{PB}$ containing $0.05 \%$ Triton $\mathrm{X}-100$ and $5 \%$ normal goat serum, the sections were incubated with the primary antibodies diluted in $0.01 \mathrm{M} \mathrm{PB}$ containing $0.05 \%$ Triton X-100 and $2 \%$ normal goat serum for $72 \mathrm{~h}$ at $4^{\circ} \mathrm{C}$ (DyLight488-Streptavidin 1:500, Jackson ImmunoResearch Laboratories; guinea pig-anti-vGLUT1 1:300, Synaptic Systems, catalog \#135 304; rabbit-anti-vGLUT2 1:250, Synaptic Systems, RRID:AB_887883; mouse-anti-vGAT 1:500, Synaptic Systems, RRID:AB_887872). After washing in $0.1 \mathrm{M} \mathrm{PB}$, the sections were incubated with the secondary antibodies diluted in $0.01 \mathrm{M} \mathrm{PB}$ containing $0.05 \%$ Triton X-100 and $2 \%$ normal goat serum overnight at $4^{\circ} \mathrm{C}$ (AlexaFluor-405 goat-anti-mouse 1:250, Invitrogen, RRID:AB_10051818; donkey-anti-guinea pig Cy3 1:300, Jackson ImmunoResearch Laboratories, catalog \#706165148; AlexaFluor-647 goat-anti-rabbit 1:200, Invitrogen, RRID:AB_1056289).

All stained sections were washed in $0.1 \mathrm{M} \mathrm{PB}$, finally incubated in $0.05 \mathrm{M}$ Tris, $\mathrm{pH}$ 8.0, for $2 \mathrm{~min}$, mounted on Superfrost glass slides, airdried overnight at $4^{\circ} \mathrm{C}$, and coverslipped with fluorescence mounting medium (Mowiol, Merck), containing 2.5\% DABCO (Sigma-Aldrich). The specificity of the primary antibodies was assessed by negative staining controls, including matched isotype controls (IgG3 isotype control antibody (Thermo Fisher Scientific; MA1-10433), used at the same concentration as the vGAT antibody and preabsorption experiments (preincubation of guinea pig-anti-vGLUT1 or rabbit-anti-vGLUT2 antibodies with 10-100 $\mu \mathrm{g}$ of the respective control peptide [Synaptic Systems; \#135-3P, \#135-4P] overnight before proceeding with the immunohistochemical staining). All negative controls revealed no staining signals, indicating a high specificity of the primary antibodies.

\section{Quantification of neuroanatomical tracings}

Histological analyses, including cell and fiber counts, as well as immunohistochemical assessments, were performed under strictly blinded conditions for all subexperiments of the study. Randomized codes were allocated to the animals after perfusion and quantifications were performed by blinded investigators. Animals with incomplete lesions in the Nissl-stained spinal cross section or with traced, descending PSN or NRG fibers running in the ipsilesional $\mathrm{C} 4-\mathrm{C} 5$ white matter were excluded from the neuroanatomical and behavioral analysis.

Cell counts in the reticular formation after retrograde C3-C4 tracing. The number of ME-positive cells was analyzed by unbiased stereological analysis on every fourth coronal brainstem section using a fluorescence microscope (10×, Axioskop 2, Zeiss). Brain sections were fitted into an atlas-based (Paxinos and Watson, 2009, RRID:nlx_152120) template using defined and prominent anatomical landmarks (facial nerve $7 \mathrm{~N}$, inferior olive and pyramidal decussation) (for detailed description, see Zörner et al., 2014), thereby normalizing the analysis to potential tissue atrophy. Retrogradely labeled cells were quantified in four regions of the reticular column: the pontine reticular nucleus, oral part $(\mathrm{PnO})$, the pontine reticular nucleus, caudal part (PnC), the NRG, and the medullary reticular nucleus, ventral part (MdV).

Quantification of double-midline crossing C3-C4 propriospinal fibers at cervical spinal segments $C 5-C 8$. Recrossing propriospinal fibers were quantified on each sixth cross-section caudal to the spinal lesion (spinal segments C5-C8). For analysis, the midline of the spinal cord was defined as the middle of the central canal. Traced propriospinal fibers intersecting this midline were defined and counted as midline recrossing C3-C4 PSN fibers. To correct for interanimal variability of tracing efficiency, absolute fiber counts of each animal were normalized to the number of traced ascending fibers in the ipsilateral white matter at cervical spinal level $\mathrm{C} 1$ of the respective animal.

Quantification of descending reticulospinal fibers rostral to the spinal lesion. NRG fiber quantification was performed by unbiased stereological analysis. To minimize fading of tracer fluorescence, each sixth crosssection of the ipsilesional spinal level C3-C4 was photographed (10X, Axioskop 2 MOT, Zeiss) and analyzed with Photoshop (Photoshop CS5). Fiber quantification was performed over a distance of $4 \mathrm{~mm}$ starting from the rostral end of the lesion (in animals $43 \mathrm{dpi}$ ) or $1 \mathrm{~mm}$ caudal to the $\mathrm{C} 3-\mathrm{C} 4$ border (in intact animals). A defined grid $(5 \times 7)$ was aligned to the boundaries of the gray matter allowing for a detailed analysis of local fiber innervation (see Fig. $2 c$ ). The proportional alignment of the grid to the gray matter allowed normalizing the analysis to potential tissue shrinkage (Tang and Nyengaard, 1997). Moreover, the stereological method enabled the analysis of lamina-specific fiber density by quantifying intersections of labeled fibers with the specific lines of the grid (Schmitz and Hof, 2005). As described previously, each intersection of a fiber with the medial and ventral boundary of a panel was taken as fiber count (Lopez et al., 2005). 3D data analysis using an assumption-based, unbiased stereological approach (West, 1999) was performed using a custom-made, automated algorithm (MATLAB R2011b, RRID:nlx_ 153890). To correct for differential tracing efficiency between animals, absolute fiber counts were normalized to the number of traced, descending reticulospinal fibers in the ipsilateral white matter at cervical level $\mathrm{C} 1$.

\section{Synaptic colocalizations and close appositions}

Colocalizations of NRG fibers with the presynaptic marker vGLUT2, as well as close appositions between NRG fibers and C3-C4 PSNs, were investigated using a spectral confocal scanning microscope (TCS SP2 AOBS, Leica Microsystems) with the lasers pretuned to $488 \mathrm{~nm}$ (ME, DyLight488), $543 \mathrm{~nm}$ (TMR), and $633 \mathrm{~nm}$ (DyLight649). For the quantification of vGLUT2/ME colocalizations, single-plane confocal images $(1024 \times 1024$ pixels; thickness of optical section: $0.53 \mu \mathrm{m})$ were acquired using a $40 \times$ oil-immersion objective (HCX PL APO Oil, NA 1.25) and $1.5 \times$ zoom at optimal antibody penetration depth for vGLUT2 (Melone et al., 2005). Quantification of the colocalizations was performed using a custom-made macro in ImageJ (Version 1.45s, National Institutes of Health, RRID:nif-0000-30467). For the representative picture of close appositions on the C3-C4 PSN, a maximal intensity projection (10 confocal images; $512 \times 512$ pixels; spaced by $0.163 \mu \mathrm{m}$ ) was acquired using a $40 \times$ oil-immersion objective and $4 \times$ zoom. Confocal images were acquired in a sequential mode to avoid crosstalk between the different channels $(488,543$, and $633 \mathrm{~nm})$. Maximum intensity and orthogonal projections of the stacks were generated using Imaris software (Version 7.4.2, Bitplane, RRID:nif-0000-00314).

Images for bouton histology were acquired using a Leica SP8 upright confocal laser scanning microscope (TCS SP8, Leica Microsystems) in 


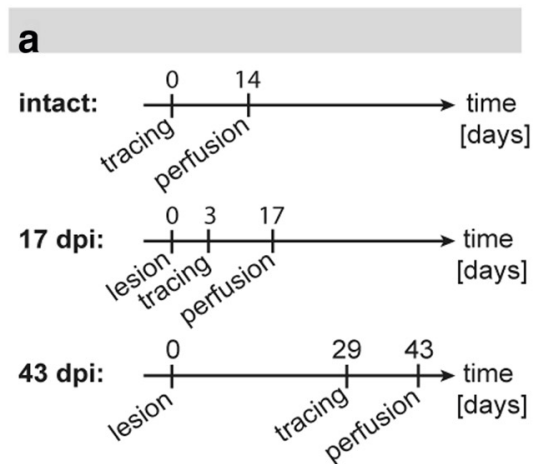

d
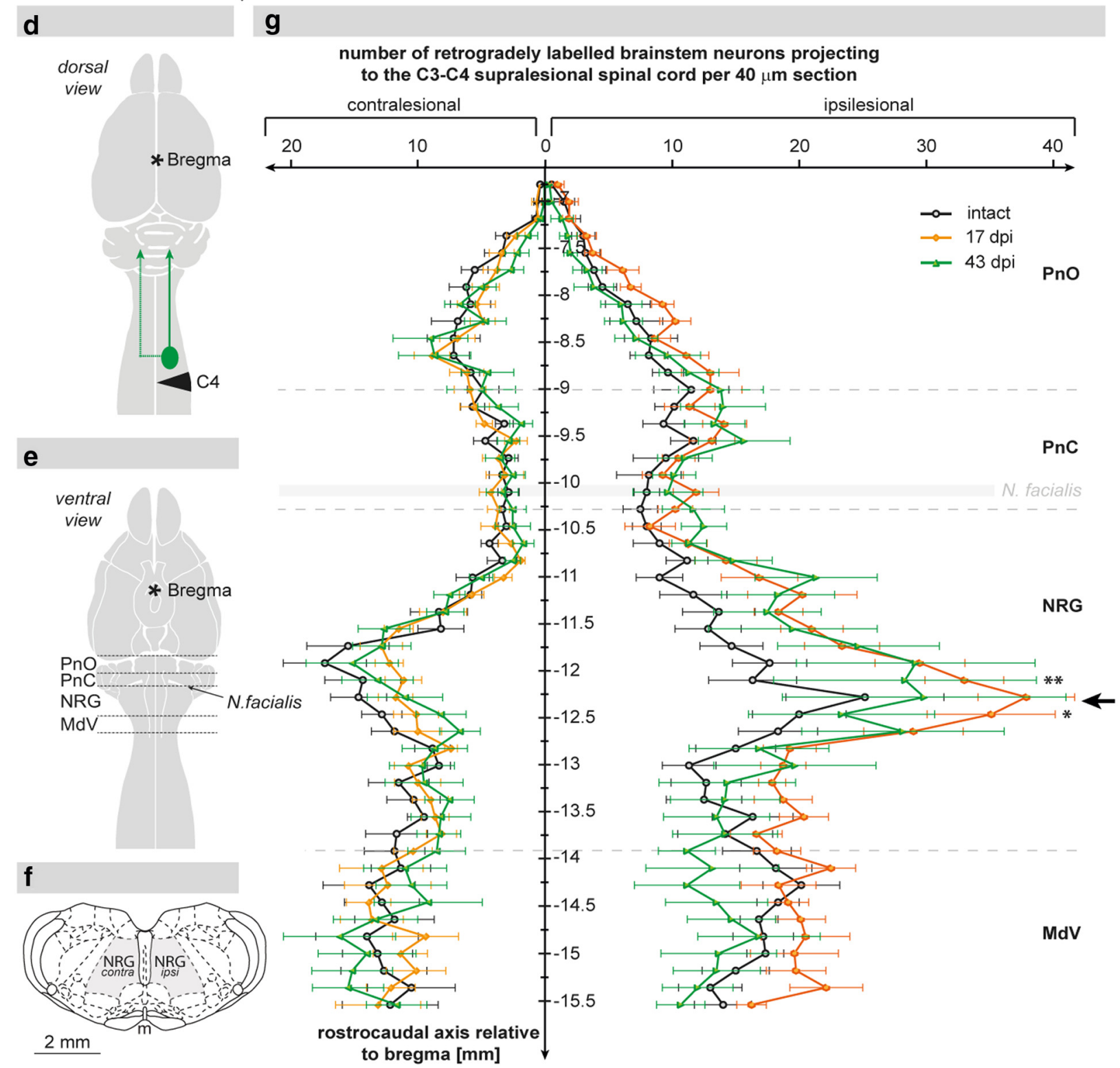

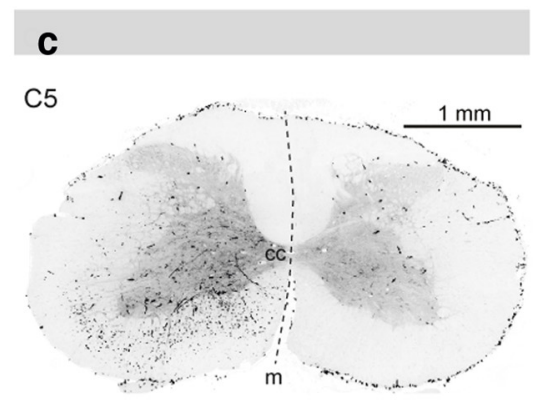

contralesional ipsilesional

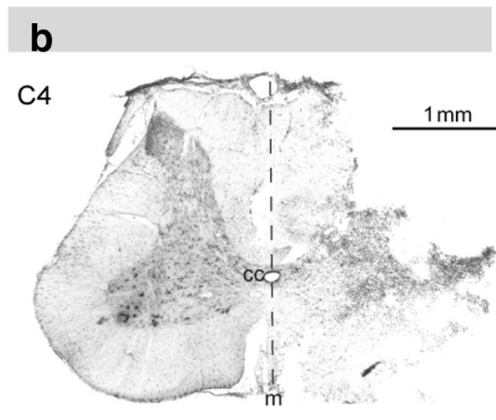

contralesional ipsilesional

Figure 1. Reticulospinal projections to the ipsilesional (3-C4 spinal cord as shown by retrograde tracing. $\boldsymbol{a}$, Experimental design displaying the time points of retrograde neuroanatomical tracing of the ipsilesional spinal segments (3-C4 and the time points of death of animals in the intact group $(n=6)$, the $17 \mathrm{~d}$ post injury (dpi) group $(n=8)$, and the $43 \mathrm{dpi}$ group $(n=6)$. $\boldsymbol{b}$, Analysis of lesion completeness: representative Nissl-stained spinal cord cross-section showing a complete right-sided C4 unilateral hemisection. c, C5 spinal cross-section of the same animal depicting the absence of labeled fibers (color-inverted) in the ipsilesional white matter below the lesion. $\boldsymbol{d}$, Scheme displaying the lesion site (black triangle), the location of tracer injection (green circle), and the route of retrograde tracer transport (green arrows). $\boldsymbol{e}$, lllustration showing the ventral view of the CNS with the regions of the reticular formation analyzed in the retrograde cell count: oral pons $(\mathrm{PnO})$, caudal pons (PnC), nucleus reticularis gigantocellularis (NRG), and medullary reticular nucleus pars ventralis (MdV). $f$, Schematic view of a coronal brainstem section displaying the reticular column (gray area) at the level of the NRG. $\boldsymbol{g}$, Quantification of retrogradely labeled neurons within the reticular formation of intact animals ( $n=6$; black), animals 17 dpi ( $n=8$; orange), and animals $43 \mathrm{dpi}(n=6$; green) per $40 \mu \mathrm{m}$ coronal brain section. Cell counts on the contralesional side were unchanged after injury, whereas the number of labeled NRG neurons on the ipsilesional side was significantly elevated 17 dpi and remained increased until $43 \mathrm{dpi}$ (black arrow). Data are group mean values \pm SEM. Data were analyzed by two-way repeated-measures ANOVA followed by post hoc Bonferroni's multiple-comparison test. ${ }^{*} p<0.05 .{ }^{* *} p<0.01$; $c c$, central canal; $m$, midline. 
the resonance mode, kindly provided by the Center for Microscopy and Image Analysis at the University of Zurich. The lasers were pretuned to $405 \mathrm{~nm}$ (goat-anti-mouse 405), $488 \mathrm{~nm}$ (ME, DyLight488), $546 \mathrm{~nm}$ (Cy3), and $647 \mathrm{~nm}$ (Cy5). For the quadruple stainings, $z$-stacks of $4-6$ $\mu \mathrm{m}$ thickness $(1024 \times 1024$ pixels, optical thickness of the section 0.17 $\mu \mathrm{m})$ were acquired in the sequential mode, using a $63 \times$ oil-immersion objective (NA 1.4), $2 \times$ zoom, and a line average of $8-16$. Confocal stacks were acquired in the optimal penetration depth of the mouse-anti-vGAT antibody, which displays the most limited penetration range into the section of the used antibodies. Quantification of marker-positive NRG varicosities was performed using the Imaris software (Version 7.4.2, Bitplane, RRID:nif-0000-00314).

\section{Locomotor assessment}

Locomotor function was quantified as described in detail previously (Zörner et al., 2010). In brief, animals were familiarized with the testing setup and trained for five sessions. Walking over a Plexiglas runway (123 $\mathrm{cm}$ long, $13 \mathrm{~cm}$ wide) was recorded with a high-speed camera $(200 \mathrm{~Hz}$, Basler A504kc Color Camera) at baseline and 7, 14, and $28 \mathrm{dpi}$. Only recording sequences with locomotor velocities between 0.2 and $0.3 \mathrm{~m} / \mathrm{s}$ and appropriate lateral stability were included for $2 \mathrm{D}$ kinematic analysis. For each animal, $>10$ representative step cycles were analyzed per time point and per locomotor parameter. The skin overlying the prominent anatomical landmarks (hindlimb: iliac crest, hip, ankle, metatarsophalangeal joint; forelimb: shoulder blade, shoulder, wrist) was tattooed (Hugo Sachs Elektronik, Harvard Apparatus), allowing permanent kinematic tracking (Clickjoint V5.0; ALEA Solutions). Maximal protraction and retraction were defined as maximal positive and negative excursion of the distal extremities (wrist and metatarsophalangeal joint) relative to the respective proximal reference points (shoulder and iliac crest). Shoulder height was defined as the vertical distance of the ipsilesional shoulder marker to the ground at the time point just before the contralesional forelimb touched the ground. At this time point, body weight support was maximally dependent on the weak, ipsilesional forelimb. The height of the iliac crest was defined as vertical distance of the ipsilesional iliac crest to the ground at the temporal midstance of the ipsilesional hindlimb. Forelimb and hindlimb dragging was defined as contact of the ipsilesional extremities during any time point of the protraction (swing) phase. Plantar paw placement was present when there was a proper contact between the plantar paw and the ground during the stance phase. Dorsal paw placement involved contact of the dorsal component of the paw with the ground during the stance phase.

\section{Statistics}

Statistical analysis was performed using SPSS (V21; SPSS, RRID: rid_000042) and GraphPad Prism (V4, GraphPad Software, RRID: rid_000081). Statistical evaluation of retrograde cell counts was performed by repeated-measures two-way ANOVA followed by post hoc Bonferroni's multiple-comparison test (see Fig. $1 \mathrm{~g}$ ). Statistical analysis of reticulospinal and propriospinal fiber counts was performed by the unpaired, two-tailed Student's $t$ test and by two-way repeated-measures ANOVA followed by post hoc Bonferroni's multiple-comparison test (see Figs. 2, 3, and 5). Statistical evaluation of behavioral data was performed by one-way repeated-measures ANOVA followed by post hoc Bonferroni's multiple-comparison test (see Fig. 6).

\section{Results}

\section{Regenerative sprouting of bulbospinal fibers rostral to the spinal injury}

Spinal cord lesions were routinely assessed in Nissl-stained spinal cord cross-sections (Fig. 1b). Moreover, the absence of traced fibers in the ipsilesional white matter next to the lesion was used to confirm lesion completeness in all animals of the study (Fig. 1c). Animals with incomplete lesions or overhemisections were excluded from the analysis. The neuroanatomical tracer ME was injected into the ipsilesional $\mathrm{C} 3-\mathrm{C} 4$ gray matter rostral to the lesion site. Retrogradely labeled neuronal somata were counted in defined brainstem areas of intact animals $(n=6)$, animals 17 dpi $(n=8)$, and animals 43 dpi $(n=6)$ (Fig. $1 a, d-g)$. In intact animals, peak numbers of cervically projecting neurons were seen at the transition of the oral $(\mathrm{PnO})$ to the caudal pons $(\mathrm{PnC})$, in the medial NRG, and in the medullary reticular nucleus pars ventralis $(\mathrm{MdV})$, with the medial NRG revealing the highest number of retrogradely labeled cells. After the injury, retrogradely labeled cells were significantly and specifically increased in the ipsilesional NRG 17 dpi (Fig. $1 g ; p<0.05$ at $12.24 \mathrm{~mm}$ relative to bregma; intact vs $17 \mathrm{dpi}$, two-way repeated-measures ANOVA followed by post hoc Bonferroni's test), and remained elevated at 43 dpi (although not statistically significant compared with intact animals). In the medial $\mathrm{PnO}$ and the caudal MdV of the ipsilesional side, cell counts were slightly elevated $17 \mathrm{dpi}$ compared with intact animals, and declined again at 43 dpi (statistically not significant). Counts of retrogradely labeled cells in the ipsilesional PnC were not altered at $17 \mathrm{dpi}$ and $43 \mathrm{dpi}$ compared with intact animals. Quantification of retrogradely labeled cells in contralesional reticular regions revealed no differences in cell numbers after injury. To specifically investigate the spinal anatomy of the descending NRG system, anterograde brainstem tracings were conducted.

\section{Fibers originating from the NRG sprout rostral to the lesion}

To quantify sprouting of severed reticulospinal fibers rostral to the lesion site, tracer was injected into the ipsilesional NRG of intact animals $(n=8)$ and animals $29 \mathrm{dpi}(n=7)$ (Fig. $1 \mathrm{~g}$, arrow; Fig. 2a). Injection sites were reconstructed for each animal (Fig. $2 b$; coordinates [mean $\pm \mathrm{SD}$ ] AP, $11.91 \pm 0.13 \mathrm{~mm}$ relative to bregma; ML, $0.9 \pm 0.1 \mathrm{~mm}$ from midline). In intact animals, NRG fibers were found to descend primarily in the ventral and lateral portion of the ipsilesional white matter, with a smaller proportion of descending fibers in the contralateral hemicord (Fig. 2d). Gigantocellular fiber innervation was highest in the intermediate and ventromedial portions of the intact, ipsilateral C3-C4 gray matter (Fig. 2d,f,g). At $43 \mathrm{~d}$ after SCI, reticulospinal fiber density was increased in the ipsilesional segments $\mathrm{C} 3-\mathrm{C} 4$ compared with the intact situation (Fig. $2 e-h$ ). The increase of NRG fiber density was observed over a distance of $3 \mathrm{~mm}$ starting $\sim 0.72 \mathrm{~mm}$ rostral to the lesion border (Fig. 2e; $p<0.0275$ for factor "injury," two-way repeated-measures ANOVA). Maximal fiber sprouting after injury was found at $3.6 \mathrm{~mm}$ distance from the rostral lesion border (Fig. $2 e ; p<0.01$, intact vs $43 \mathrm{dpi}$, post hoc Bonferroni's test). Lesion-induced arborization of reticulospinal fibers was highly region-specific, with the medial and ventral C3-C4 gray matter disclosing an increased fiber density at 43 dpi (Fig. 2f; column 3; $p<0.05$; Fig. $2 g$, rows 6 and 7; $p<0.001$ and $p<0.01$, two-way repeated-measures ANOVA with post hoc Bonferroni's test). Region-specific fiber sprouting was confirmed by a heat plot displaying the difference in NRG fiber density between 43 dpi and the intact situation (Fig. $2 h$ ): enhanced fiber density 43 dpi was primarily found in the ventromedial gray matter corresponding to laminae VII-IX of the ipsilesional segments C3-C4. The most medial and lateral areas as well as the complete dorsal half of the gray matter did not reveal an enhanced NRG fiber innervation (Fig. $2 h$ ). To further assess the innervation of the supralesional segments by descending gigantocellular fibers, we quantified the number of gigantocellular fibers crossing the ipsilesional white-gray matter transition at level C3-C4. The number of fibers entering the ipsilesional C3-C4 gray matter was not different between intact and spinal cord-injured animals (Fig. $2 i ; p=0.791$; intact vs $43 \mathrm{dpi}$, Student's unpaired $t$ test). This implies that fiber arborization within specific areas of the gray matter rather 


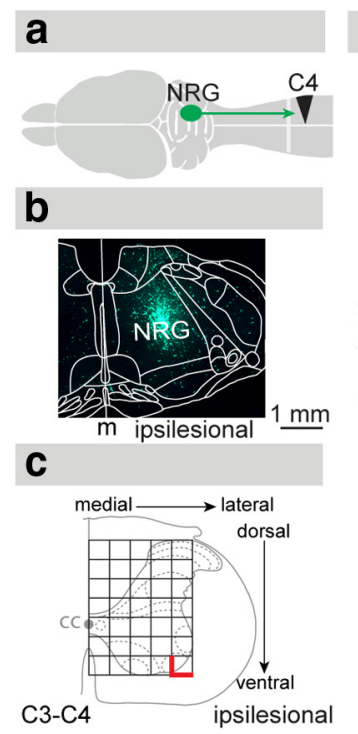

\section{d}

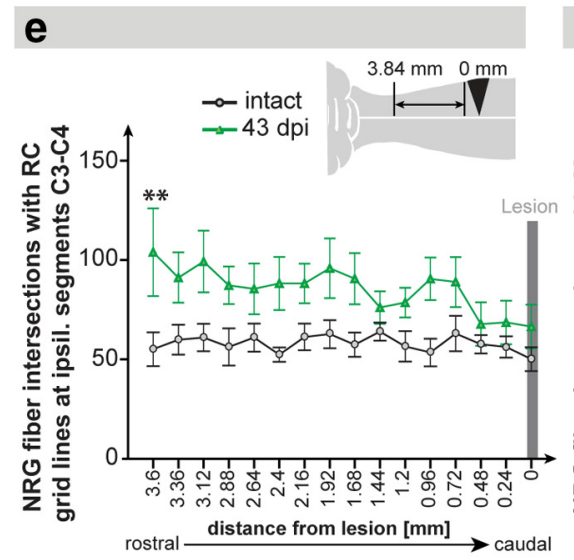

\section{h}

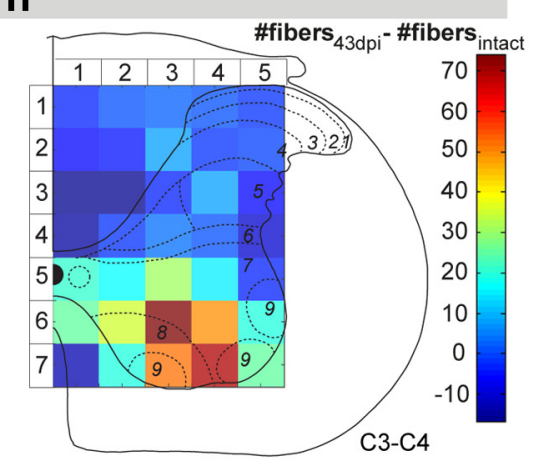

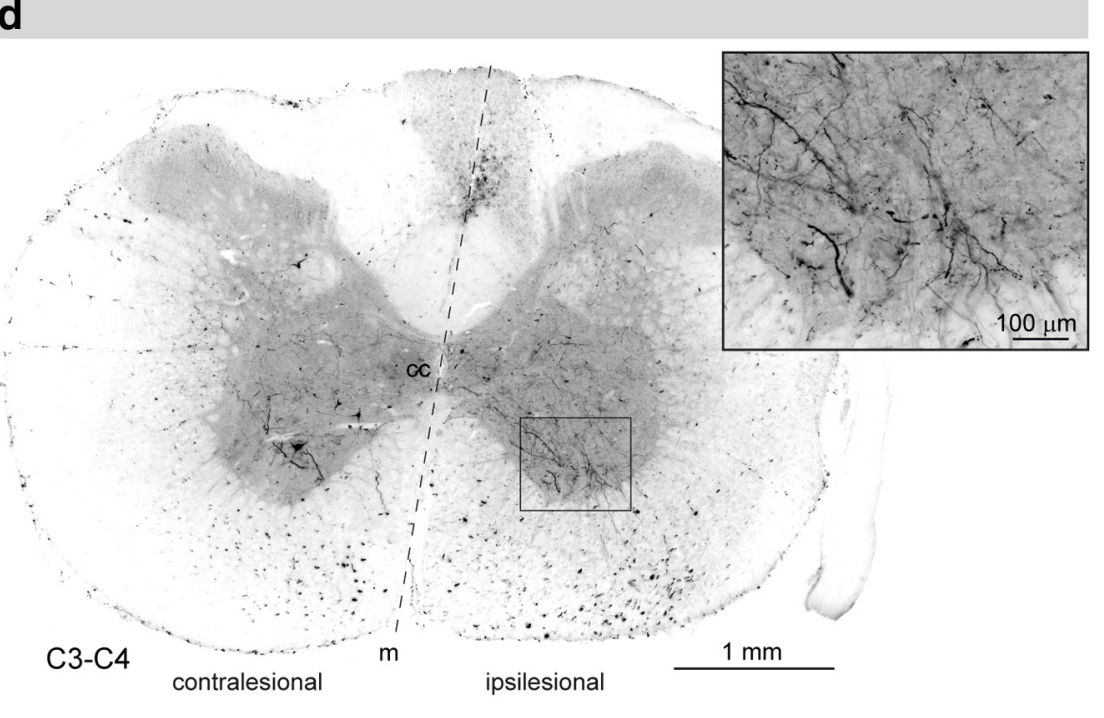
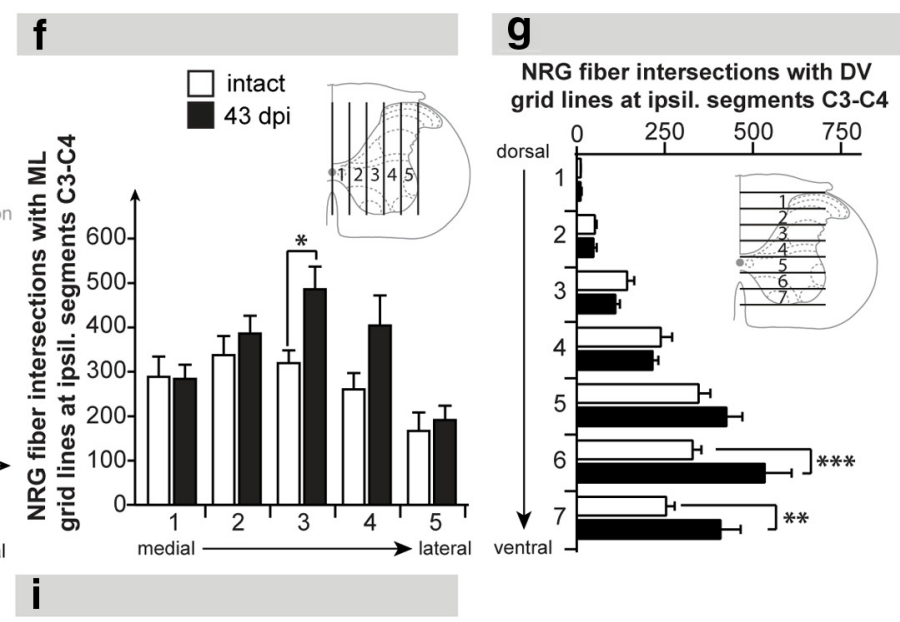

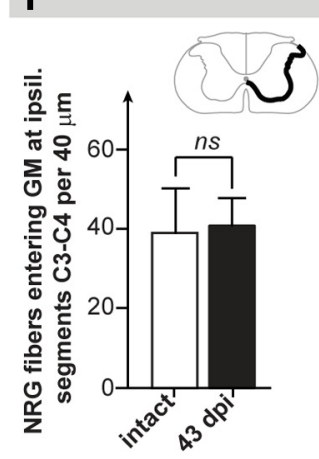

Figure 2. Anterograde tracing of reticulospinal fibers originating from the ipsilesional NRG. $\boldsymbol{a}$, Schematic illustration showing the site of anterograde tracer injection (ipsilesional NRG), the anterograde tracer transport, and the lesion site. $\boldsymbol{b}$, Representative injection site of the tracer ME in the ipsilesional NRG. c, Scheme displaying the $5 \times 7$ grid used for stereological quantification of reticulospinal fiber density at the ipsilesional C3-C4 spinal cord. Intersections of axons with the medial and ventral lines (bold red lines) of each rectangle were defined as fiber counts. $\boldsymbol{d}$, Representative (3-C4 cross-section showing labeled descending NRG fibers at 43 dpi primarily in the ventromedial and ventral funiculi of the ipsilateral white matter innervating the ventral horn of the gray matter (inset). $\boldsymbol{e}$, Quantification of gigantocellular fibers along the rostrocaudal (RC) axis in the ipsilesional C3-C 4 gray matter. Fiber counts were augmented $43 \mathrm{dpi}$ (green line) compared with intact animals (gray line). $f$, Quantification of reticulospinal fibers along the mediolateral axis in the ipsilesional (3-C4 gray matter. Fiber counts were specifically increased in the central part of the gray matter $43 \mathrm{dpi}$ (black bars) compared with intact animals (white bars). $\boldsymbol{g}$, Quantification of NRG fibers along the dorsoventral axis in the ipsilesional C3-C4 gray matter. Fiber counts were specifically increased in the most ventral gray matter 43 dpi compared with intact animals. $\boldsymbol{h}$, Heat plot illustrating the difference in fiber counts between $43 \mathrm{dpi}$ and the intact situation. NRG fiber density was primarily increased in the ventromedial gray matter of the ipsilesional segments C3-C4. $\boldsymbol{i}$, Collaterals of descending NRG fibers per $40 \mu \mathrm{m}$ cross-section entering the spinal gray matter (GM) at ipsilesional spinal segments (3-C4. Fiber counts were not different in animals $43 \mathrm{dpi}$ and intact animals. Data points $(\boldsymbol{e})$ and bars $(\boldsymbol{f}, \boldsymbol{g}, \boldsymbol{i})$ represent group mean values \pm SEM. Analysis of groups was performed by two-way repeated-measures ANOVA followed by post hoc Bonferroni's multiple-comparison test. Comparison of total mean values between intact and $43 \mathrm{dpi}$ was performed by the unpaired, two-tailed Student's $t$ test. ${ }^{*} p<$ $0.05 .{ }^{* *} p<0.01$. cc, central canal; contral, contralesional; $m$, midline; ipsil, ipsilesional; $n s$, not significant. 

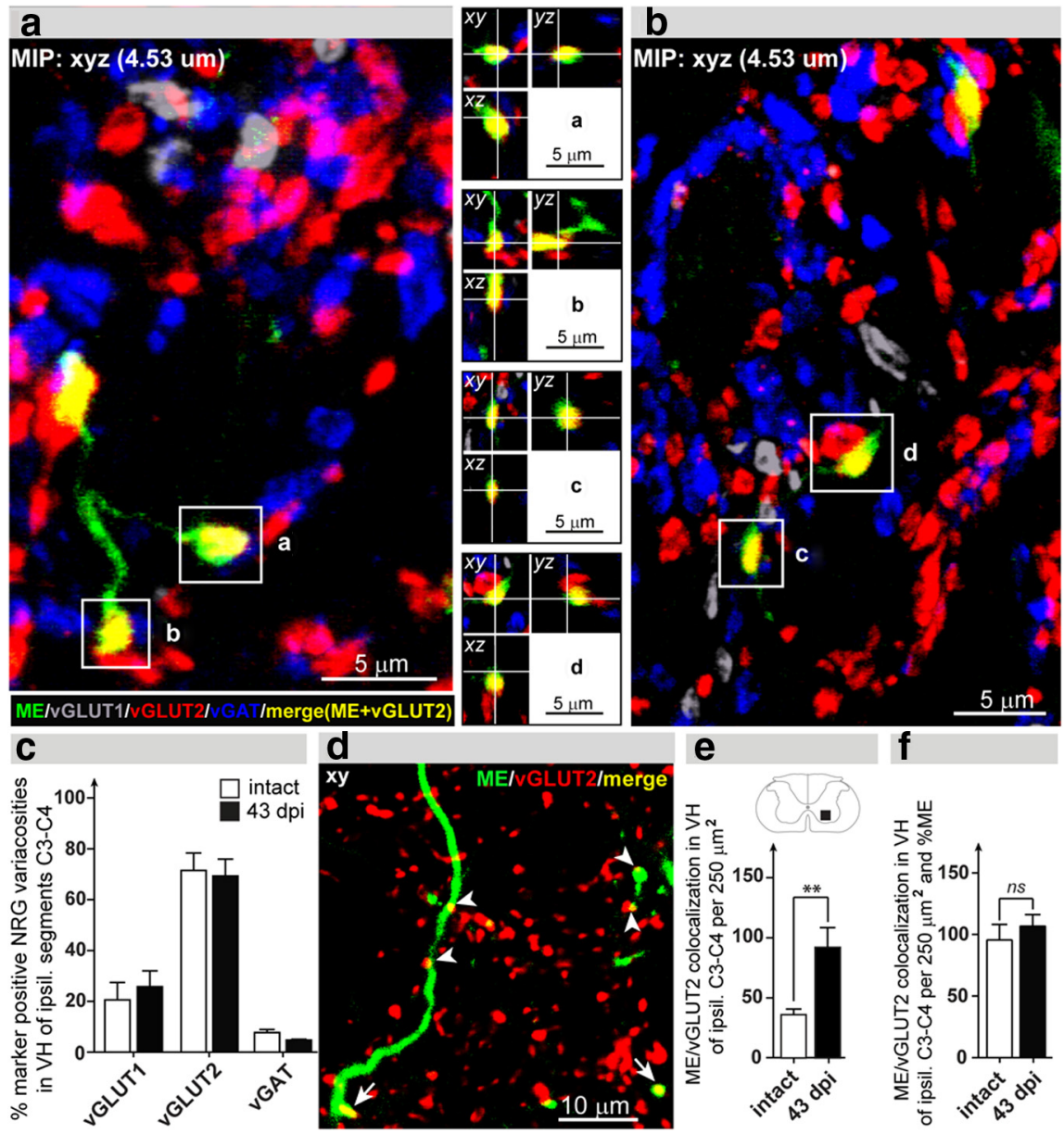

Figure 3. Synaptic integration of NRG fibers rostral to the injury. $\boldsymbol{a}, \boldsymbol{b}$, High-resolution $(63 \times)$ pictures showing maximum intensity projections (MIP) with quadruple staining: green represents ME; gray represents VGLUT1; red represents VGLUT2; blue represents vGAT. ME-traced NRG fibers (green) form vGLUT2-positive varicosities (yellow) ( $\boldsymbol{a}$ - $\boldsymbol{d}$, insets with orthogonal views). $\boldsymbol{c}$, $3 D$ analysis of synaptic neurotransmitter phenotype revealed that NRG fibers primarily form vGLUT2-postive synapses. The proportion of the neurotransmitter phenotype is unchanged after injury. $\boldsymbol{d}$, Representative confocal image (optical thickness 0.53 $\mu \mathrm{m}$ ) from the ipsilesional C3-C4 ventral horn (VH). ME-positive NRG fibers (green) display several colocalizations (merge: yellow) with vGLUT2 (red). $\boldsymbol{e}$, Number of colocalizations between ME-positive gigantocellular fibers and vGLUT2-positive varicosities per $250 \mu \mathrm{m}^{2}$ in VH of the ipsilesional segments (3-C4. Colocalizations were more than doubled in spinal cord-injured animals. $f$ Colocalizations of NRG fibers with vGLUT2 patches normalized for the total ME signal per area of investigation. vGLUT2-positive varicosities per ME-positive pixels were not different between animals at $43 \mathrm{dpi}$ and intact animals. $\boldsymbol{c}, \boldsymbol{e}, \boldsymbol{f}$, Error bars indicate group mean values \pm SEM. Comparison of total mean values between intact and $43 \mathrm{dpi}$ was performed by the unpaired, two-tailed Student's $t$ test. ${ }^{*} p<0.05 .{ }^{* *} p<0.01$. ns, not significant.

than increased collateral sprouting of descending NRG fibers into the gray matter primarily accounts for the significantly increased NRG fiber density after SCI.

Reticulospinal fibers display vGLUT2-positive varicosities, which increase in number after injury

To investigate the synaptic integration of NRG fibers in the cervical spinal cord rostral to the lesion site, we examined colocalizations of the anterogradely labeled ME-positive reticulospinal fibers with the presynaptic markers vGLUT1, vGLUT2, and vGAT. Characterization of synaptic transmitter phenotypes was performed by high-resolution confocal analysis in intact animals $(n=3)$ and animals 43 dpi $(n=3$; Fig. $3 a-c)$. For each varicosity, a 3D analysis of confocal image stacks was performed to assure that the synaptic markers are within the ME-positive NRG bouton rather than in an adjacent structure (e.g., a bouton) ending on the ME-positive NRG fiber (Fig. 3a,b, insets). NRG fiber varicosities were predominantly vGLUT2-positive in intact $(71.6 \%$ of all pre-

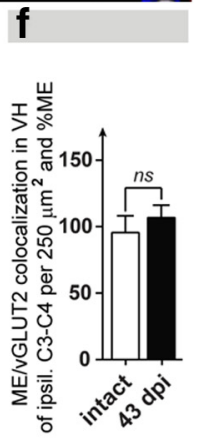

synaptic colocalizations) and injured animals (69.4\%; Fig. 3c). vGLUT1-positive (intact, 20.6\%; 43 dpi, 25.8\%) and vGATpositive (intact, $7.8 \%$; $43 \mathrm{dpi}, 4.8 \%$ ) presynaptic colocalizations were less abundant, which is in agreement with previous results showing that glutamate (in particular the vGLUT2 system) represents the predominant transmitter system in brainstem neurons responsible for excitatory motor commands (Hägglund et al., 2010). The proportion of marker-positive NRG varicosities did not alter after SCI (Fig. 3c). vGLUT2-positive colocalizations were found in "en passant" (Fig. 3b, inset, $d$ ) and in terminal boutons (Fig. $3 a, b$ insets, $a-c$ ).

In a next step, we quantified the total number of ME/vGLUT2-positive colocalizations per $250 \mu \mathrm{m}^{2}$ in the ipsilesional C3-C4 ventral horn of intact $(n=8)$ and chronically lesioned $(n=7)$ animals. Quantification of ME/vGLUT2 colocalizations was performed in single-plane confocal images (optical thickness, 0.53 $\mu \mathrm{m})$. VGLUT2-positive NRG varicosities were more than doubled after SCI (Fig. $3 e$; $p=0.0068$, Student's unpaired $t$ test). When the number of colocalizations was normalized to the total ME-signal per 250 $\mu \mathrm{m}^{2}$, there was only a minor difference between spinal cord-injured and intact animals (Fig. 3f; $p=0.489$; intact vs 43 dpi, Student's unpaired $t$ test). These results suggest that the new gigantocellular fibers form synapses at a similar density, such as the preexisting fibers.

\section{Reticulospinal fibers form close} appositions with descending, doublemidline crossing $\mathrm{C} 3-\mathrm{C} 4$ propriospinal neurons

To unveil cellular targets of the descending NRG fibers rostral to the lesion, the retrograde tracer TMR was injected into the ipsilesional cervical spinal cord below the lesion (spinal segments C6-C8) (Fig. 4a,b). At the level of the lesion, labeled axons were exclusively present in the ventral white matter of the contralesional hemicord, but not in the destroyed and scarred white matter of the lesioned C4 hemicord, thus confirming the completeness of the hemisections. Labeled propriospinal neurons were mainly found in ventromedial areas of the ipsilesional gray matter rostral to the lesion. The axons of these cells must have crossed the spinal cord midline twice: above the lesion to the intact hemicord and below the lesion to innervate the injected ipsilesional segments $\mathrm{C} 6-\mathrm{C} 8$. Interestingly, their cell body location correlates with the area of maximal NRG fiber density at 43 dpi (Fig. 4c).

Simultaneously with the retrograde tracing from the ipsilesional segments C6-C8 with TMR, the ipsilesional NRG was anterogradely traced with ME to assess possible contacts between descending reticulospinal fibers and descending, double-midline crossing PSNs. Close appositions between descending, ME-positive fibers and TMR-positive PSNs were frequently found on dendrites and on so- 
mata (Fig. 4d). Acquisition of confocal stacks allowed for 3D analysis of close appositions, thereby demonstrating the rewiring of gigantocellular fibers onto lesionbypassing C3-C4 PSNs (Fig. 4d).

Increased innervation of the denervated lower cervical spinal cord by recrossing projections from $\mathrm{C} 3-\mathrm{C} 4$ propriospinal neurons

Upon tracer injection into the ipsilesional spinal segment C3-C4, ME-positive fibers were quantified in the cervical segments C5-C8 of animals at $17 \mathrm{dpi}(n=8)$ and 43 dpi $(n=6)$ (Fig. $5 a)$. C3-C4 PSN fibers were seen to cross the spinal cord midline above the lesion site and then descended scattered within the contralesional white matter (Fig. 5b), as described for propriospinal fibers (Reed et al., 2006; Flynn et al., 2011). Quantification of descending C3-C4 PSN fibers in the contralesional white matter of spinal segment C5 revealed no difference between animals 17 dpi and 43 dpi (Fig. $5 c ; p=0.7603 ; 17 \mathrm{dpi}$ vs $43 \mathrm{dpi}$, Student's unpaired $t$ test). The number of descending PSN fibers entering the gray matter in the contralesional segments C6-C8, however, was substantially increased at 43 dpi compared with 17 dpi (Fig. $5 d ; p=0.0027$, Student's unpaired $t$ test). This enhanced collateralization and innervation of the sublesional cervical spinal cord were statistically significant across all cervical segments C6-C8 (Fig. $5 e ; p=0.0029$ for factor "time after injury"; 17 dpi vs $43 \mathrm{dpi}$; two-way repeated-measures ANOVA). Most importantly, some of these fibers crossed the spinal cord midline to innervate the denervated gray matter of the segments below the injury. The number of PSN fibers segmentally recrossing to the ipsilesional side was more than doubled at 43 dpi compared with 17 dpi (Fig. $5 f$; $p=0.0101$, Student's unpaired $t$ test). The increase of midlinerecrossing C3-C4 PSN fibers after injury was observed across the cervical segments C6-C8 (Fig. $5 g ; p=0.0109$ for factor "time after injury," two-way repeated-measures ANOVA) and was more pronounced in caudal than rostral cervical segments. Bridging PSN fibers mainly projected to the medial portions of the intermediate and ventral horn of the ipsilesional cervical enlargement (Rexed's laminae VII and VIII), which are areas primarily containing premotor interneurons (Fig. 5h).

\section{Locomotor recovery after cervical unilateral hemisection}

Longitudinal assessment of locomotor function was performed before the injury (baseline $[\mathrm{BL}])$ and 7,14 , and $28 \mathrm{dpi}(n=6$ animals). Cervical transection of the right hemicord caused an extensive initial impairment (BL vs $7 \mathrm{dpi}$ ) of the ipsilesional forelimb and of both hindlimbs (Fig. 6). The hindlimbs subsequently showed profound locomotor recovery from 7 to $28 \mathrm{dpi}$. In contrast, the ipsilesional forelimb remained severely impaired displaying a reduced range of motion persisting over 4 weeks. The maximal forward position (protraction) of the wrist relative to the shoulder was significantly decreased at 7 dpi (Fig. $6 a ; p<$ 0.001; BL vs $7 \mathrm{dpi}$; one-way repeated-measures ANOVA with post hoc Bonferroni's test; for all data in Fig. 6) and remained impaired
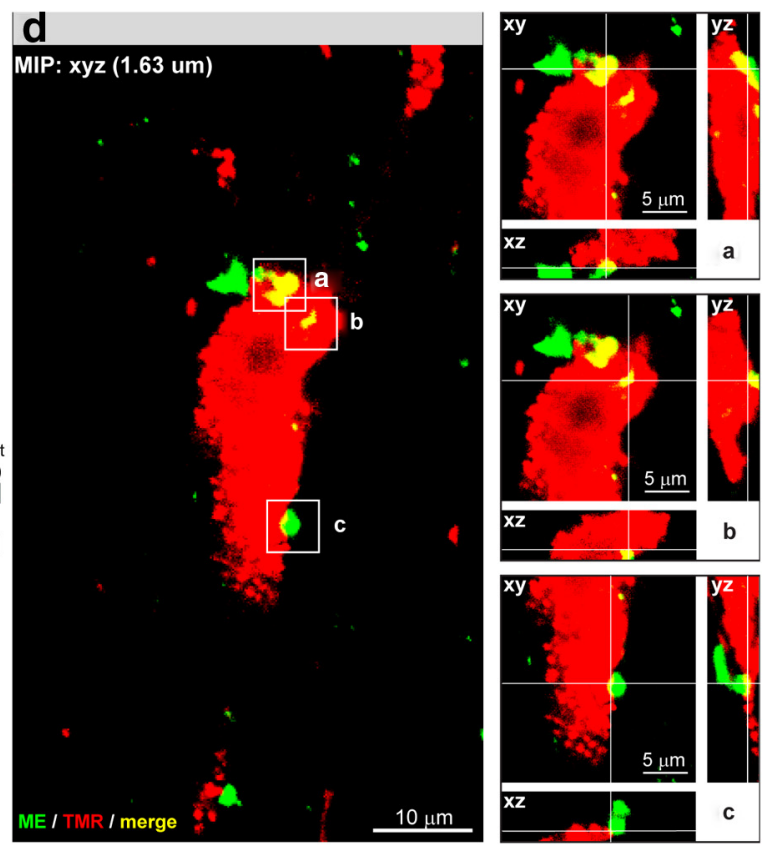

Figure 4. Close appositions of reticulospinal fibers onto descending, double-midline crossing C3-C4 PSNs. $\boldsymbol{a}$, Scheme illustrating the (he ipsilesional NRG (anterograde, ME) and the C3-C4 PSNs projecting to the ipsilesional spinal segments C6 -C8 in the background. The location of double-midline crossing PSNscorrelates with the region of maximal gigantocellularfibe close NRG fiber appositions on the cell depicted in $\boldsymbol{d}$ at high resolution.

up to $28 \mathrm{dpi}$. The maximal backward position (retraction) of the ipsilesional forelimb was slightly increased $7 \mathrm{dpi}$ and did not recover until $28 \mathrm{dpi}$ (Fig. $6 a$ ). Shoulder height of the ipsilesional forelimb was not affected by the lesion and showed baseline levels at $28 \mathrm{dpi}$ (Fig. 6b). Proper paw clearance of the ipsilesional forelimb was abolished after injury and showed no signs of improvement until 28 dpi (Fig. 6e; BL: 100\% steps without paw dragging; 7-28 dpi: 0\% steps without paw dragging). In contrast, correct paw placement of the ipsilesional forelimb improved from $34 \%$ plantar steps (of total steps) at $7 \mathrm{dpi}$ to $90 \%$ plantar steps at $28 \mathrm{dpi}$ (Fig. 6f; $p<0.05,7$ dpi vs $28 \mathrm{dpi}$ ).

Protraction and retraction of the ipsilesional hindlimb revealed significant lesion deficits acutely after injury (Fig. $6 c$; protraction: $p<0.001$, BL vs 7 dpi; retraction: $p<0.01$, BL vs 7 dpi) but showed significant functional recovery almost achieving baseline performance at $28 \mathrm{dpi}$ (protraction: $p<0.01,7 \mathrm{dpi}$ vs 28 dpi; retraction: $p<0.05$, 7 dpi vs $28 \mathrm{dpi}$ ). The height of the ipsilesional iliac crest was not changed after injury. The trend toward a reduced crest height 7 dpi compared with the intact situation probably indicates a reduced weight support of the ipsilesional hindlimb acutely after injury. The ipsilesional iliac crest height returned to baseline level at $28 \mathrm{dpi}$ (Fig. $6 d$ ). Paw clearance of the ipsilesional hindlimb was substantially deteriorated after injury: Paw dragging of the ipsilesional hindlimb was persistent and omnipresent over the first 2 weeks (Fig. $6 g ; p<0.001$, BL vs $7 \mathrm{dpi}$ ) and was mostly observed at the initial swing phase. However, in contrast to the persistent paw dragging of the ipsilesional forelimb, the ipsilesional hindlimb significantly improved its paw clearance up to 4 weeks after SCI (Fig. $6 g ; p<0.01,7$ dpi vs 28 dpi). Paw placement of the ipsilesional hindlimb was not impaired by the SCI throughout the experiment (Fig. 6h). 

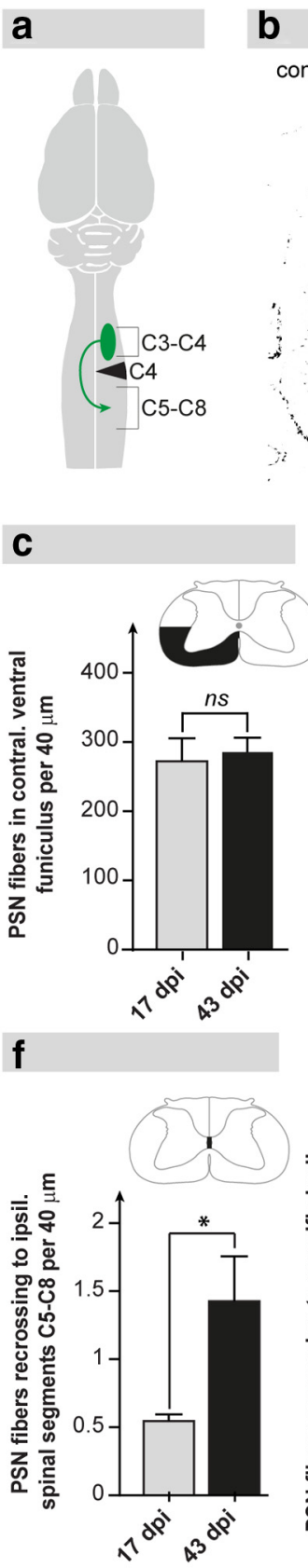

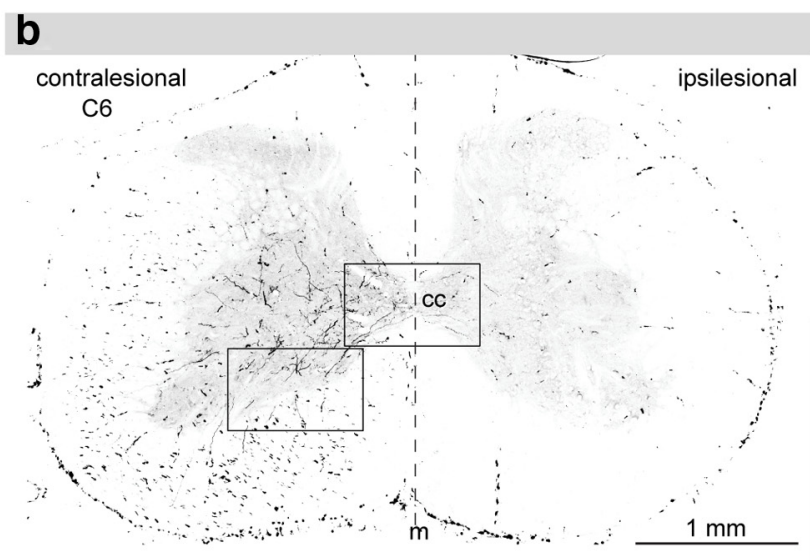

d

e
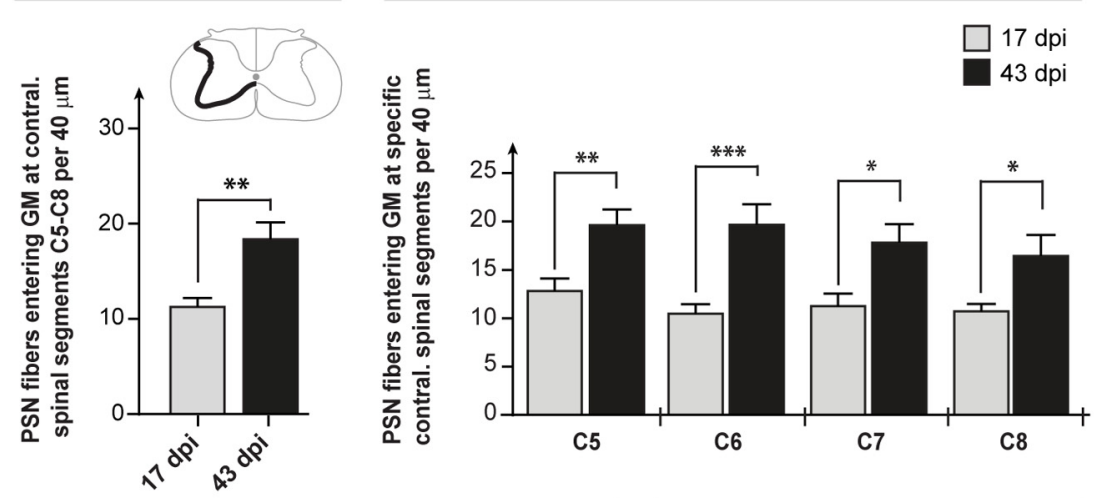

9
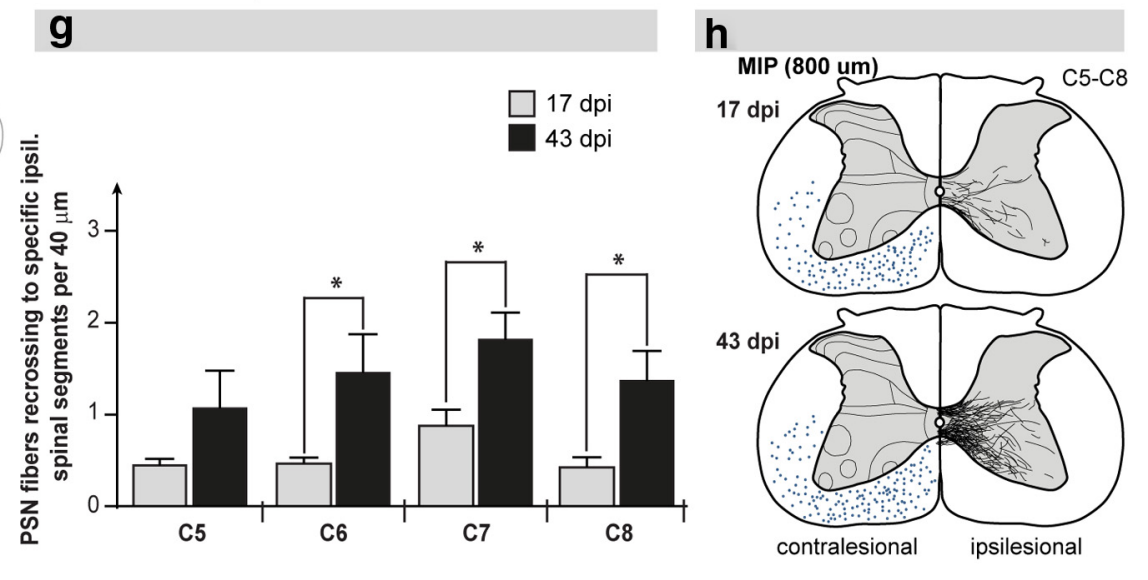

Figure 5. Descending (3-C4 PSN axons sprout and recross the spinal cord midline below the injury. $\boldsymbol{a}$, Scheme illustrating the anterograde tracer injections in the ipsilesional spinal segments C3-C4 and the area of fiber quantification in the cervical enlargement (C6-C8). $\boldsymbol{b}$, Representative picture displaying C3-C4 PSN fibers at spinal level C6 at 43 dpi. The fibers enter the contralesional C6 gray matter (top inset, red arrows) and a portion of them recross over the midline ( $m$ ) to innervate the sublesional denervated hemicord (bottom inset, red arrows). $c$, Numbers of descending PSN fibers in the ventral white matter at level C5 (black area) per $40 \mu \mathrm{m}$ cross-section were not different between animals at $17 \mathrm{dpi}(n=8)$ and $43 \mathrm{dpi}(n=6)$. $\boldsymbol{d}$, The number of descending PSN fibers

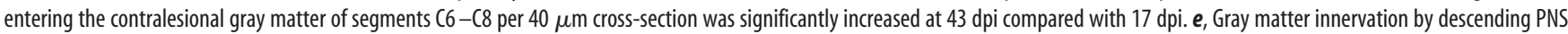
collaterals per $40 \mu \mathrm{m}$ cross-section across cervical segments C 6 - 8 was enhanced across all cervical segments. $f$, Axons of C3-C4 PSNs recross the spinal cord midline below the lesion. Recrossing PSN fibers were more than doubled at $43 \mathrm{dpi} \mathrm{compared} \mathrm{with} 17 \mathrm{dpi} . \boldsymbol{g}$, Midline recrossing PSN fibers per $40 \mu \mathrm{m}$ cross-section were increased across all cervical segments (C6 -C8) at $43 \mathrm{dpi}$. $\boldsymbol{h}$, Camera lucida overlays (20 cross-sections) showing recrossing PSN fibers in segments (6-C 8 of one representative animal at $17 \mathrm{dpi}$ (top) and at $43 \mathrm{dpi}$ (bottom). $\boldsymbol{e}, \boldsymbol{g}$, Analysis of segments was subjected to two-way repeated-measures ANOVA followed by post hoc Bonferroni's multiple-comparison test. Comparison of total mean values between $17 \mathrm{dpi}$ and $43 \mathrm{dpi}$ was performed by the unpaired, two-tailed Student's $t$ test. ${ }^{*} p<0.05 .{ }^{* *} p<0.01 .{ }^{* *} p<0.001$. cc, central canal; m, midline; GM, gray matter; MIP, maximum intensity projection; VH, ventral horn; $n s$, not significant.

\section{Discussion}

Our results demonstrate a remarkable capacity of severed reticulospinal fibers to sprout above a spinal cord hemisection and to form excitatory boutons, some of which end on C3-C4 propriospinal neurons. Axons of these cervical PSNs passed the lesion site in the spared hemicord and sprouted below the lesion across the spinal cord midline into the ventral horn of the denervated hemi- cord. This neuroanatomical bypass around the lesion was accompanied by a significant restoration of locomotor function.

Severed reticulospinal fibers sprout and form vGLUT2positive varicosities rostral to the lesion

Retrograde tracing data revealed differential plastic responses among bulbospinal systems to SCI. The NRG was the only reticu- 
lospinal system displaying a substantial increase in retrogradely labeled cell bodies following SCI, indicating enhanced sprouting and arborization of transsected NRG axons rostral to the injury. Other reticulospinal systems revealed no or a minor sprouting response after injury. A reason for the differential plastic responses among the reticulospinal systems might be dissimilar intrinsic, growth-related properties (e.g., expression of regeneration-associated proteins, response to trophic factors) (Plunet et al., 2002). Moreover, the distance of the soma to the lesion site (Fernandes et al., 1999) and the neural activity within a certain system (Luo and O'Leary, 2005) might influence the cellular response to axonal damage. Absolute cell counts of the different descending brainstem systems and the laterality of their spinal projections in intact animals were in accordance with earlier studies (Sakai et al., 2009). Anterograde tracer injection into the ipsilesional NRG demonstrated a significant, lesioninduced sprouting and arborization of reticulospinal fibers in the ventromedial C3-C4 gray matter (layers VII-IX) above the lesion. The pronounced sprouting response of NRG fibers seen in the anterograde versus retrograde data might be explained by the differential analysis (fibers vs somata) and different efficiencies and transport mechanisms of the tracers (Rajakumar et al., 1993). The distribution of sprouting NRG fibers corresponds to the reticulospinal termination pattern found in intact animals (Matsuyama et al., 2004; Ballermann and Fouad, 2006), implying the existence of mechanisms that either attract regrowing fibers to the correct target zones or prune aberrantly grown fibers.

NRG fibers in the C3-C4 ventral horn formed primarily vGLUT2-positive varicosities ( 70\%); vGLUT1-positive ( 25\%) and vGAT-positive boutons $(\sim 5 \%)$ were less frequent. Analysis of neurotransmitter phenotypes was performed by highresolution, 3D confocal microscopy, assuring proper colocalization of the presynaptic markers with ME-traced axonal structures. vGLUT2-positive NRG varicosities were in the size of synaptic boutons and were present as "en-passant" and as terminal boutons. Quantification of vGLUT2/ME colocalizations was performed in single-plane confocal images with small optical thickness $(0.53 \mu \mathrm{m})$. The number of vGLUT2-positive NRG varicosities per area was more than doubled in the C3-C4 ventral gray matter of chronically injured rats ( $43 \mathrm{dpi}$ ) compared with the intact cord. These results imply a substantial increase in excitatory output from the ipsilateral NRG to the supralesional spinal segments after SCI. geal joint.
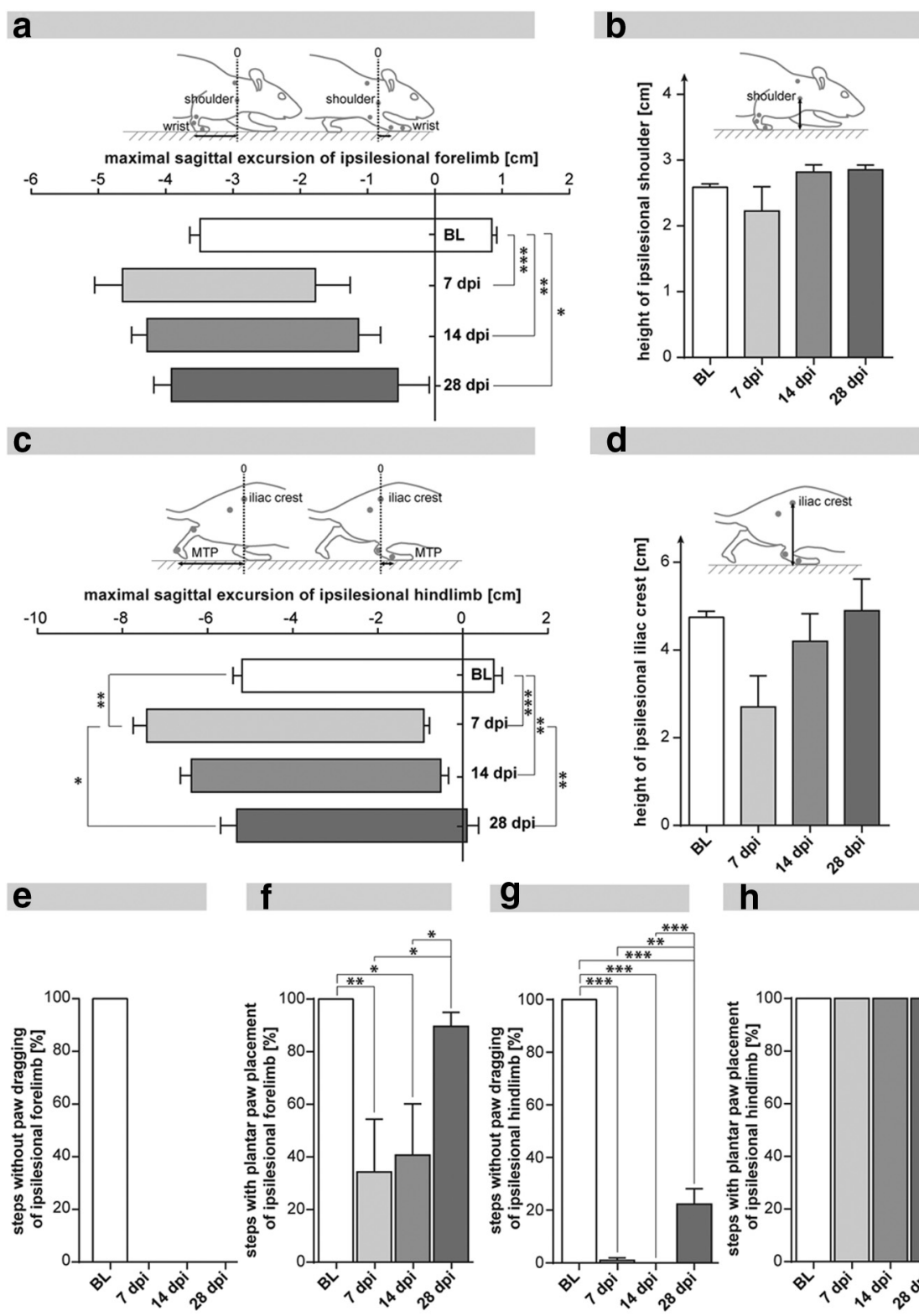

h

Figure 6. Locomotor impairment and recovery after unilateral $C 4$ hemisection in adult rats. $\boldsymbol{a}$, Protraction and retraction of the ipsilesional forelimb relative to the shoulder marker revealed substantial lesion deficits, which did not recover up to $28 \mathrm{dpi}$. $\boldsymbol{b}$, The shoulder height of the ipsilesional forelimb was mildly impaired after injury and showed complete functional recovery until $28 \mathrm{dpi}$. c, Protraction and retraction of the ipsilesional hindlimb relative to the iliac crest marker showed a significant lesion deficit, which was followed by significant restoration of hindlimb flexion and extension up to $28 \mathrm{dpi}$. $\boldsymbol{d}$, The height of the ipsilesional iliac crest was reduced to half acutely after injury and recovered up to baseline levels 28 dpi. $\boldsymbol{e}$, Animals showed persistent dragging of the ipsilesional forelimb without any recovery until $28 \mathrm{dpi}$. $\boldsymbol{f}$, The proportion of plantar steps of the ipsilesional forelimb recovered significantly after the initial impairment at 7 dpi. $\boldsymbol{g}$, Ipsilesional hindlimb dragging was omnipresent until 14 dpi and showed incomplete, but significant, functional recovery at $28 \mathrm{dpi}$. $\boldsymbol{h}$, Plantar stepping of the ipsilesional hindlimb was not impaired by the spinal injury. Graphs represent group mean values \pm SEM. Data were subjected to one-way repeated-measures ANOVA followed by post hoc Bonferroni's multiple-comparison test. ${ }^{*} p<0.05$. ${ }^{* *} p<0.01$. ${ }^{* *} p<0.001$. BL, baseline; MTP, metatarsophalan-

So far, regenerative sprouting of axotomized reticulospinal fibers in higher mammals was only observed after treatments with growth factor cocktails and plasticity-enhancing substances (García-Alías et al., 2011; Lu et al., 2012) or into peripheral nerve grafts (Richardson et al., 1984). After incomplete lesions of the lower thoracic cord, Ballermann and Fouad (2006) observed spontaneous sprouting of spared reticulospinal fibers below the lesion, but no sprouting of the thoracically injured fibers at the cervical level. 


\section{Reticulospinal fibers contact C3-C4 propriospinal neurons that bridge the lesion site}

In the present study, we concentrated on double-midline crossing propriospinal projections, which have been previously reported for descending cervical PSNs (Reed et al., 2006). Remarkably, the somata of recrossing C3-C4 PSNs were found primarily in the ventral horn area with the highest NRG fiber density at 43 dpi (laminae VII-IX) and corresponded to the previously described location of descending cervical PSNs (Bareyre et al., 2004, Reed et al., 2006). 3D confocal stack analysis disclosed frequent close appositions between gigantocellular fibers and somata or proximal dendrites of double-crossing PSNs, suggesting the formation of synaptic contacts. Monosynaptic innervation of C3-C4 PSNs by reticulospinal fibers was found in intact cats (Illert et al., 1981), arguing for a reinforcement of preexisting reticulo-propriospinal connections following injury in the present experiments.

The formation of spontaneous propriospinal detours that reroute supraspinal commands around a spinal lesion has been described previously. PSNs above a thoracic hemisection revealed the capacity to relay supraspinal input around staggered thoracic hemisections to the lumbar cord, thereby restoring some locomotor functions in mice (Courtine et al., 2008). Other studies demonstrated that relaying PSNs were contacted by severed corticospinal fibers, which triggered locomotor improvements in rats with incomplete SCI (Bareyre et al., 2004; van den Brand et al., 2012). A series of experiments in cats and primates investigated the physiology of the $\mathrm{C} 3-\mathrm{C} 4$ propriospinal relay under normal and pathological circumstances. Propriospinal transmission of cortical commands is essential for the control of hand dexterity in intact primates (Kinoshita et al., 2012). After incomplete cervical SCI, axotomized corticospinal fibers synapse onto C3-C4 PSNs, which innervate cervical motoneurons (Blagoveshchenskii et al., 2005; Alstermark et al., 2011). This intraspinal relay was associated with significant recovery of reaching and digit movements after injury. In healthy humans, cortical commands to upper extremity motoneurons are partially transmitted through C3-C4 PSNs (Marchand-Pauvert et al., 2001). In patients with cortical stroke, supraspinal transmission through the C3-C4 relay is enhanced on the hemiplegic side, suggesting a potential contribution of propriospinal relays to motor recovery also in humans (Pierrot-Deseilligny, 2002). An analogous C3-C4 propriospinal system in rodents has not been described yet, however.

\section{C3-C4 propriospinal axons recross the midline and enhance their projections to the denervated spinal cord}

The number of descending C3-C4 fibers entering the contralesional C6-C8 gray matter was almost doubled at 43 dpi compared with $17 \mathrm{dpi}$ : $8 \%$ of these fibers recrossed the spinal cord midline to the ipsilesional, denervated gray matter at $43 \mathrm{dpi}$. These back-crossing propriospinal fibers could enhance the indirect access of reticulospinal commands to the denervated cord. Plasticity of double-midline crossing propriospinal projections has been associated with functional recovery after incomplete SCI previously (Courtine et al., 2008; Schnell et al., 2011), implying the importance of these projections for recovery after SCI. Somata of double-crossing C3-C4 neurons were mainly localized in the ventromedial horn, which is similar to previous findings on descending C3-C4 PSNs projecting to segments C6-C8 in cats and monkeys (Alstermark et al., 1990; Kinoshita et al., 2012). Double-crossing fibers could contact cervical premotor interneurons and medial motoneurons, possibly explaining the pronounced recovery of the proximal (shoulder and elbow) forelimb of the hemisected rats in the present study. Some of these fibers may also relay to long descending PSNs projecting to lumbar spinal segments, the somata of which are primarily localized in laminae VII-IX (Vavrek et al., 2006).

\section{Recovery of locomotor function}

Functional recovery after cervical unilateral hemisection was characterized by significant recovery of hindlimb functions and limited recovery of the ipsilesional forelimb, which is in agreement with previous findings (Webb and Muir, 2002; Filli et al., 2011). Compensatory sprouting of spared descending systems was associated with functional recovery after unilateral hemilesions (Rosenzweig et al., 2010). Interestingly, Zörner et al. (2014) showed that restricted electrolytic microlesion of the ipsilesional NRG resulted in the reappearance of significant deficits of forelimb and hindlimb locomotion in chronic C4 hemisected rats. The recovered weight support of the ipsilesional forelimb as well as the improved hindpaw clearance and step width seen at 4 months after SCI significantly worsened $2 \mathrm{~d}$ after ipsilesional NRG ablation. The absence of functional deficits after unilateral NRG lesions in intact animals points to a functional role of the plastic alterations shown in the present study. This interpretation is in line with previous concepts on the role of the $\mathrm{C} 3-\mathrm{C} 4$ propriospinal system for motor recovery in cats, monkeys, and humans (Pierrot-Deseilligny, 2002; Alstermark et al., 2007). However, functional ablation of alternative pathways originating from the ipsilesional NRG (e.g., intrabulbar connections) cannot be excluded by microlesions. Further experiments (e.g., transsynaptic tracing, optogenetics) are required to conclusively disclose the physiological relevance of the C3-C4 PSN bypass after spinal hemisection.

In conclusion, the present study shows a remarkable level of spontaneous adaptive fiber growth and bouton formation by reticulospinal and propriospinal axons following incomplete SCI in adult rats. Reticulospinal fiber density doubled in the ventral parts of the spinal segments rostral to the lesion. NRG fibers formed predominantly vGLUT2-positive varicosities; their numbers doubled in the segments rostral to the injury, and they formed close appositions onto descending C3-C4 propriospinal neurons. The axons of these propriospinal neurons in turn increased the innervation density in the ipsilesional and contralesional spinal cord below the lesion. Behavioral recovery and lesion data indicate a potential significance of this reticulopropriospinal detour pathway for locomotor recovery. Given the high relevance of the reticulospinal system not only for basic motor functions, such as locomotion and posture (Peterson, 1979), but also for skilled forelimb movements in monkeys (Soteropoulos et al., 2012) and humans (Honeycutt et al., 2013), the high plastic potential of this system is intriguing and makes it an important target for future interventions aiming to restore motor function after SCI.

\section{References}

Alstermark B, Isa T, Kümmel H, Tantisira B (1990) Projection from excitatory C3-C4 propriospinal neurones to lamina VII and VIII neurones in the C6Th1 segments of the cat. Neurosci Res 8:131-137. CrossRef Medline

Alstermark B, Pinter MJ, Sasaki S (1992) Descending pathways mediating disynaptic excitation of dorsal neck motoneurones in the cat: brain stem relay. Neurosci Res 15:42-57. CrossRef Medline

Alstermark B, Isa T, Pettersson LG, Sasaki S (2007) The C3-C4 propriospinal system in the cat and monkey: a spinal pre-motoneuronal centre for voluntary motor control. Acta Physiol (Oxf) 189:123-140. CrossRef Medline

Alstermark B, Pettersson LG, Nishimura Y, Yoshino-Saito K, Tsuboi F, Takahashi M, Isa T (2011) Motor command for precision grip in the ma- 
caque monkey can be mediated by spinal interneurons. J Neurophysiol 106:122-126. CrossRef Medline

Ballermann M, Fouad K (2006) Spontaneous locomotor recovery in spinal cord injured rats is accompanied by anatomical plasticity of reticulospinal fibers. Eur J Neurosci 23:1988-1996. CrossRef Medline

Bareyre FM, Kerschensteiner M, Raineteau O, Mettenleiter TC, Weinmann O, Schwab ME (2004) The injured spinal cord spontaneously forms a new intraspinal circuit in adult rats. Nat Neurosci 7:269-277. CrossRef Medline

Blagoveshchenskii ED, Pettersson LG, Perfil'ev SN (2005) Control of fine movements mediated by propriospinal neurons. Neurosci Behav Physiol 35:299-304. CrossRef Medline

Courtine G, Song B, Roy RR, Zhong H, Herrmann JE, Ao Y, Qi J, Edgerton VR, Sofroniew MV (2008) Recovery of supraspinal control of stepping via indirect propriospinal relay connections after spinal cord injury. Nat Med 14:69-74. CrossRef Medline

Fernandes KJ, Fan DP, Tsui BJ, Cassar SL, Tetzlaff W (1999) Influence of the axotomy to cell body distance in rat rubrospinal and spinal motoneurons: differential regulation of GAP-43, tubulins, and neurofilament-M. J Comp Neurol 414:495-510. CrossRef Medline

Filli L, Zörner B, Weinmann O, Schwab ME (2011) Motor deficits and recovery in rats with unilateral spinal cord hemisection mimic the BrownSequard syndrome. Brain 134:2261-2273. CrossRef Medline

Flynn JR, Graham BA, Galea MP, Callister RJ (2011) The role of propriospinal interneurons in recovery from spinal cord injury. Neuropharmacology 60:809-822. CrossRef Medline

García-Alías G, Petrosyan HA, Schnell L, Horner PJ, Bowers WJ, Mendell LM, Fawcett JW, Arvanian VL (2011) Chondroitinase ABC combined with neurotrophin NT-3 secretion and NR2D expression promotes axonal plasticity and functional recovery in rats with lateral hemisection of the spinal cord. J Neurosci 31:17788-17799. CrossRef Medline

Gerasimenko Y, Roy RR, Edgerton VR (2008) Epidural stimulation: comparison of the spinal circuits that generate and control locomotion in rats, cats and humans. Exp Neurol 209:417-425. CrossRef Medline

Hägglund M, Borgius L, Dougherty KJ, Kiehn O (2010) Activation of groups of excitatory neurons in the mammalian spinal cord or hindbrain evokes locomotion. Nat Neurosci 13:246-252. CrossRef Medline

Honeycutt CF, Kharouta M, Perreault EJ (2013) Evidence for reticulospinal contributions to coordinated finger movements in humans. J Neurophysiol 110:1476-1483. CrossRef Medline

Illert M, Jankowska E, Lundberg A, Odutola A (1981) Integration in descending motor pathways controlling the forelimb in the cat: 7. Effects from the reticular formation on $\mathrm{C} 3-\mathrm{C} 4$ propriospinal neurones. Exp Brain Res 42:269-281. CrossRef Medline

Kapitza S, Zörner B, Weinmann O, Bolliger M, Filli L, Dietz V, Schwab ME (2012) Tail spasms in rat spinal cord injury: changes in interneuronal connectivity. Exp Neurol 236:179-189. CrossRef Medline

Kinjo N, Atsuta Y, Webber M, Kyle R, Skinner RD, Garcia-Rill E (1990) Medioventral medulla-induced locomotion. Brain Res Bull 24:509-516. CrossRef Medline

Kinoshita M, Matsui R, Kato S, Hasegawa T, Kasahara H, Isa K, Watakabe A, Yamamori T, Nishimura Y, Alstermark B, Watanabe D, Kobayashi K, Isa $\mathrm{T}$ (2012) Genetic dissection of the circuit for hand dexterity in primates. Nature 487:235-238. CrossRef Medline

Lawrence DG, Kuypers HG (1968) The functional organization of the motor system in the monkey: II. The effects of lesions of the descending brain-stem pathways. Brain 91:15-36. CrossRef Medline

Lopez I, Ishiyama G, Tang Y, Frank M, Baloh RW, Ishiyama A (2005) Estimation of the number of nerve fibers in the human vestibular endorgans using unbiased stereology and immunohistochemistry. J Neurosci Methods 30:145:37-46. CrossRef Medline

Lu P, Wang Y, Graham L, McHale K, Gao M, Wu D, Brock J, Blesch A, Rosenzweig ES, Havton LA, Zheng B, Conner JM, Marsala M, Tuszynski MH (2012) Long-distance growth and connectivity of neural stem cells after severe spinal cord injury. Cell 150:1264-1273. CrossRef Medline

Luo L, O'Leary DD (2005) Axon retraction and degeneration in development and disease. Annu Rev Neurosci 28:127-156. CrossRef Medline

Marchand-Pauvert V, Mazevet D, Pradat-Diehl P, Alstermark B, PierrotDeseilligny E (2001) Interruption of a relay of corticospinal excitation by a spinal lesion at C6-C7. Muscle Nerve 24:1554-1561. CrossRef Medline

Matsuyama K, Mori F, Nakajima K, Drew T, Aoki M, Mori S (2004) Locomotor role of the corticoreticular-reticulospinal-spinal interneuronal system. Prog Brain Res 143:239-249. CrossRef Medline
Melone M, Burette A, Weinberg RJ (2005) Light microscopic identification and immunocytochemical characterization of glutamatergic synapses in brain sections. J Comp Neurol 492:495-509. CrossRef Medline

Noga BR, Kriellaars DJ, Brownstone RM, Jordan LM (2003) Mechanism for activation of locomotor centers in the spinal cord by stimulation of the mesencephalic locomotor region. J Neurophysiol 90:1464-1478. CrossRef Medline

Paxinos G, Watson C (2009) The rate brain in stereotaxic coordinates, Ed 6. San Diego: Academic.

Peterson BW (1979) Reticulospinal projections to spinal motor nuclei. Annu Rev Physiol 41:127-140. CrossRef Medline

Pierrot-Deseilligny E (2002) Propriospinal transmission of part of the corticospinal excitation in humans. Muscle Nerve 26:155-172. CrossRef Medline

Plunet W, Kwon BK, Tetzlaff W (2002) Promoting axonal regeneration in the central nervous system by enhancing the cell body response to axotomy. J Neurosci 68:1-6. CrossRef Medline

Rajakumar N, Elisevich K, Flumerfelt BA (1993) Biotinylated dextran: a versatile anterograde and retrograde neuronal tracer. Brain Res 607:47-53. CrossRef Medline

Reed WR, Shum-Siu A, Onifer SM, Magnuson DS (2006) Inter-enlargement pathways in the ventrolateral funiculus of the adult rat spinal cord. Neuroscience 142:1195-1207. CrossRef Medline

Richardson PM, Issa VM, Aguayo AJ (1984) Regeneration of long spinal axons in the rat. J Neurocytol 13:165-182. CrossRef Medline

Rosenzweig ES, Courtine G, Jindrich DL, Brock JH, Ferguson AR, Strand SC, Nout YS, Roy RR, Miller DM, Beattie MS, Havton LA, Bresnahan JC, Edgerton VR, Tuszynski MH (2010) Extensive spontaneous plasticity of corticospinal projections after primate spinal cord injury. Nat Neurosci 13:1505-1510. CrossRef Medline

Sakai ST, Davidson AG, Buford JA (2009) Reticulospinal neurons in the pontomedullary reticular formation of the monkey (Macaca fascicularis). Neuroscience 163:1158-1170. CrossRef Medline

Schmitz C, Hof PR (2005) Design-based stereology in neuroscience. Neuroscience 130:813-831. CrossRef Medline

Schnell L, Hunanyan AS, Bowers WJ, Horner PJ, Federoff HJ, Gullo M, Schwab ME, Mendell LM, Arvanian VL (2011) Combined delivery of Nogo-A antibody, neurotrophin-3 and the NMDA-NR2d subunit establishes a functional 'detour' in the hemisected spinal cord. Eur J Neurosci 34:1256-1267. CrossRef Medline

Shik ML, Orlovsky GN (1976) Neurophysiology of locomotor automatism. Physiol Rev 56:465-501. Medline

Soteropoulos DS, Williams ER, Baker SN (2012) Cells in the monkey pontomedullary reticular formation modulate their activity with slow finger movements. J Physiol 590:4011-4027. CrossRef Medline

Tang Y, Nyengaard JR (1997) A stereological method for estimating the total length and size of myelin fibers in human brain white matter. J Neurosci Methods 73:193-200. CrossRef Medline

Tator CH, Duncan EG, Edmonds VE, Lapczak LI, Andrews DF (1995) Neurological recovery, mortality and length of stay after acute spinal cord injury associated with changes in management. Paraplegia 33:254-262. CrossRef Medline

van den Brand R, Heutschi J, Barraud Q, DiGiovanna J, Bartholdi K, Huerlimann M, Friedli L, Vollenweider I, Moraud EM, Duis S, Dominici N, Micera S, Musienko P, Courtine G (2012) Restoring voluntary control of locomotion after paralyzing spinal cord injury. Science 336:1182-1185. CrossRef Medline

Vavrek R, Girgis J, Tetzlaff W, Hiebert GW, Fouad K (2006) BDNF promotes connections of corticospinal neurons onto spared descending interneurons in spinal cord injured rats. Brain 129:1534-1545. CrossRef Medline

Webb AA, Muir GD (2002) Compensatory locomotor adjustments of rats with cervical or thoracic spinal cord hemisections. J Neurotrauma 19: 239-256. CrossRef Medline

West MJ (1999) Stereological methods for estimating the total number of neurons and synapses: issues of precision and bias. Trends Neurosci 22: 51-61. CrossRef Medline

Zörner B, Schwab ME (2010) Anti-Nogo on the go: from animal models to a clinical trial. Ann N Y Acad Sci 1198 [Suppl 1]:22-34.

Zörner B, Filli L, Starkey ML, Gonzenbach R, Kasper H, Röthlisberger M, Bolliger M, Schwab ME (2010) Profiling locomotor recovery: comprehensive quantification of impairments after CNS damage in rodents. Nat Methods 7:701-708. CrossRef Medline

Zörner B, Bachmann LC, Filli L, Kapitza S, Gullo M, Bolliger M, Starkey ML, Röthlisberger M, Gonzenbach RR, Schwab ME (2014) Chasing CNS plasticity: the brainstem's contribution to locomotor recovery in spinal cord injured rats. Brain 137:1716-1732. CrossRef Medline 